\section{The AFSUMB Consensus Statements and Recommendations for the Clinical Practice of Contrast-Enhanced Ultrasound using Sonazoid}

\author{
Jae Young Lee ${ }^{1 *}$, Yasunori Minami ${ }^{2 *}$, Byung Ihn Choi ${ }^{3}$, Won Jae Lee ${ }^{4}$, Yi-Hong Chou ${ }^{5,6}$, \\ Woo Kyoung Jeong ${ }^{4}$, Mi-Suk Park ${ }^{7}$, Nobuki Kudo ${ }^{8}$, Min Woo Lee ${ }^{4}$, Ken Kamata ${ }^{2}$, \\ Hiroko lijima ${ }^{9}$, So Yeon Kim ${ }^{10}$, Kazushi Numata ${ }^{11}$, Katsutoshi Sugimoto ${ }^{12}$, \\ Hitoshi Maruyama ${ }^{13}$, Yasukiyo Sumino ${ }^{14}$, Chikara Ogawa ${ }^{15}$, Masayuki Kitano ${ }^{16}$, ljin Joo', \\ Junichi Arita ${ }^{17}$, Ja-Der Liang ${ }^{18}$, Hsi-Ming Lin ${ }^{19}$, Christian Nolsoe ${ }^{20}$, Odd Helge Gilja ${ }^{21,22}$, \\ Masatoshi Kudo ${ }^{2}$
}

*Author affiliations appear at the end of this article.

The first edition of the guidelines for the use of ultrasound contrast agents was published in 2004, dealing with liver applications. The second edition of the guidelines in 2008 reflected changes in the available contrast agents and updated the guidelines for the liver, as well as implementing some nonliver applications. The third edition of the contrast-enhanced ultrasound (CEUS) guidelines was the joint World Federation for Ultrasound in Medicine and BiologyEuropean Federation of Societies for Ultrasound in Medicine and Biology (WFUMB-EFSUMB) venture in conjunction with other regional US societies such as Asian Federation of Societies for Ultrasound in Medicine and Biology, resulting in a simultaneous duplicate on liver CEUS in the official journals of both WFUMB and EFSUMB in 2013. However, no guidelines were described mainly for Sonazoid due to limited clinical experience only in Japan and Korea. The new proposed consensus statements and recommendations provide general advice on the use of Sonazoid and are intended to create standard protocols for the use and administration of Sonazoid in hepatic and pancreatobiliary applications in Asian patients and to improve patient management.

Keywords: Pancreatobiliary; Sonazoid; Contrast media; Consensus; Liver; Ultrasonography

\section{Introduction}

With increased use of Sonazoid (Daiichi-Sankyo, Tokyo, Japan; GE Healthcare, Milwaukee, WI, USA) in Asian countries, the need for Asian guidelines on the use of contrast-enhanced ultrasound (CEUS) using Sonazoid has become apparent. The Korean Society of Ultrasound in Medicine initiated discussions to create guidelines or consensus statements for Sonazoid in CEUS with the Japanese Society of Ultrasound in Medicine in 2019, in conjunction with the Chinese Taipei Society of Ultrasound in Medicine, recognizing the fact that ultrasound contrast agents (UCAs) are now licensed

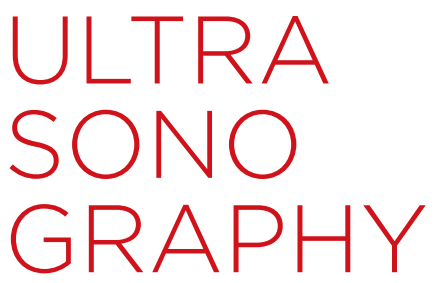

\section{GUIDELINE}

https://doi.org/10.14366/usg.20057 pISSN: 2288-5919 - elSSN: 2288-5943 Ultrasonography 2020;39:191-220

Received: April 20, 2020

Revised: April 20, 2020

Accepted: April 27, 2020

Correspondence to:

Byung Ihn Choi, MD, Department of Radiology, Chung Ang University Hospital, 84 Heukseok-ro, Dongjak-gu, Seoul 06974, Korea

Tel. +82-2-6299-3204

Fax. +82-2-6299-2064

E-mail: bichoi@caumc.or.kr

Masatoshi Kudo, MD, Department of Gastroenterology and Hepatology, Faculty of Medicine, Kindai University, Higashi-Osaka, Japan

Tel. +81-72-366-0221 (ext. 3149)

Fax.+81-72-367-2880

E-mail: m-kudo@med.kindai.ac.jp

* These authors contributed equally to this work.

This guideline is being published concurrently in Journal of Medical Ultrasound 2020;28:59-82. Copyright holder of this guideline is Journal of Medical Ultrasound (the official journal of Asian Federation of Societies for Ultrasound in Medicine).

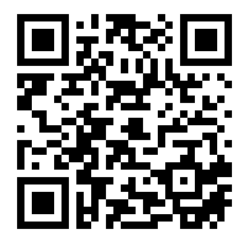

How to cite this article:

Lee JY, Minami Y, Choi BI, Lee WJ, Chou YH, Jeong WK, et al. The AFSUMB Consensus Statements and Recommendations for the Clinical Practice of Contrast-Enhanced Ultrasound using Sonazoid. Ultrasonography. 2020 Jul;39(3):191-220. 
in Asian regions of the world, including Japan, Korea, Taiwan, and China.

To produce a set of new CEUS hepatobiliary guidelines or consensus statements, two meetings of 23 representatives and experts from three Asian countries (Japan, Korea, and Taiwan) were held in Korea in April and in Japan in May 2019 (Table 1). Although a significant portion of the work was accomplished at the meeting, the group continued to meet via conference calls and at local meetings.

These consensus statements and recommendations are based on comprehensive literature surveys, including results from prospective clinical trials. On topics for which no significant clinical study data were available, evidence was obtained from expert committee reports or was based on the consensus of experts in the field of ultrasound (US) and CEUS during the consensus meetings. Quality of evidence and strength of recommendations were assigned according to the Grading of Recommendations, Assessment, Development, and Evaluation (GRADE) system [1] for quality of evidence: high $(A)$, moderate $(B)$, and low $(C)$ and for strength of recommendation: strong (1) and weak (2). The quality of evidence and strength of each recommendation were established by vote based on the criteria of the GRADE system. In addition, all consensus statements were rated by all participants on a scale of 10 ( 1 lowest to 10 highest) and averaged to show the agreement level, overall evidence level, and recommendation level in numbers.

These consensus statements and recommendations provide general advice on the use of Sonazoid. They may be used to create standard protocols for the use and administration of Sonazoid in hepatic and pancreatobiliary applications in Asian patients and to improve patient management.

\section{Table 1. Contents and author contributions}

\begin{tabular}{ll}
\hline \multicolumn{1}{c}{ Section } & Responsibility \\
\hline Introduction & Byung Ihn Choi \\
General considerations & \\
1. History & Byung Ihn Choi \\
& Masatoshi Kudo \\
& Christian Nolsoe \\
& Odd Helge Gilja \\
2. Basics & Nobuki Kudo \\
3. Terminology & ljin Joo \\
& Yasunori Minami \\
Chapter 1. Technical aspects & Yi-Hong Chou \\
1. Pharmacokinetics & \\
& Hiroko lijima \\
2. Imaging techniques & Jae Young Lee \\
\hline
\end{tabular}

Table 1. Continued

\begin{tabular}{|c|c|}
\hline Section & Responsibility \\
\hline & Kazushi Numata \\
\hline & Yi-Hong Chou \\
\hline \multicolumn{2}{|l|}{ Chapter 2. Detection of focal liver lesions } \\
\hline \multirow[t]{3}{*}{ 1. Focal hepatic lesions } & Masatoshi Kudo \\
\hline & Mi-Suk Park \\
\hline & Ja-Der Liang \\
\hline \multirow[t]{2}{*}{ 2. Intraoperative ultrasound } & Junichi Arita \\
\hline & Min Woo Lee \\
\hline \multicolumn{2}{|l|}{ Chapter 3. Characterization of focal liver lesions } \\
\hline \multirow[t]{2}{*}{ 1. In noncirrhotic liver } & Woo Kyoung Jeong \\
\hline & Katsutoshi Sugimoto \\
\hline \multirow[t]{3}{*}{ 2. In cirrhotic liver } & Yasunori Minami \\
\hline & Woo Kyoung Jeong \\
\hline & Ja-Der Liang \\
\hline \multirow[t]{2}{*}{ 3. Portal vein thrombosis } & Hitoshi Maruyama \\
\hline & ljin Joo \\
\hline 4. Macroscopic classification & Yasunori Minami \\
\hline \multicolumn{2}{|l|}{ Chapter 4. Guiding intervention } \\
\hline 1. Biopsy & So Yeon Kim \\
\hline \multirow[t]{4}{*}{ 2. Interventional tumor ablation } & Min Woo Lee \\
\hline & Yasunori Minami \\
\hline & Chikara Ogawa \\
\hline & Hsi-Ming Lin \\
\hline \multicolumn{2}{|l|}{ Chapter 5. Quantitative CEUS } \\
\hline \multirow[t]{4}{*}{ 1. Monitoring treatment response } & Chikara Ogawa \\
\hline & So Yeon Kim \\
\hline & Yasunori Minami \\
\hline & Hsi-Ming Lin \\
\hline \multirow[t]{2}{*}{ 2. Others (fibrosis, etc.) } & Yasukiyo Sumino \\
\hline & Woo Kyoung Jeong \\
\hline \multicolumn{2}{|l|}{ Chapter 6. Other organs } \\
\hline 1. CEUS for pancreatobiliary disease & Hitoshi Maruyama \\
\hline 2. Contrast-enhanced EUS for pancreas & Masayuki Kitano \\
\hline 3. Contrast-enhanced EUS for gallbladder & Ken Kamata \\
\hline \multicolumn{2}{|l|}{ Chapter 7. Others } \\
\hline \multirow[t]{2}{*}{ 1. Limitations } & Jae Young Lee \\
\hline & Hiroko lijima \\
\hline \multirow[t]{2}{*}{ 2. Artifacts } & Jae Young Lee \\
\hline & Hitoshi Maruyama \\
\hline \multirow[t]{3}{*}{ 3. Safety considerations } & Nobuki Kudo \\
\hline & Mi-Suk Park \\
\hline & Yi-Hong Chou \\
\hline 4. Contraindications & So Yeon Kim \\
\hline
\end{tabular}

CEUS, contrast-enhanced ultrasound; EUS, endoscopic ultrasound. 


\section{General Considerations}

\section{History \\ Contrast-enhanced ultrasound and ultrasound contrast agents}

One of the first reports on the use of CEUS was in 1969, by Gramiak and Shah, who performed echocardiography after injection of agitated saline into a patient's bloodstream. Since then, many UCAs have been developed, including Albunex, Echovist, Levovist, Definity, Sonovist, Echogen, Optison, SonoVue, Imagent, Quantison, and Sonazoid.

Among these, the UCAs most popular in Asia for liver imaging are Levovist, SonoVue, and Sonazoid. Levovist is a first-generation UCA and is no longer used. SonoVue is widely available in Europe and Asia and recently in North America under the trade name of Lumason. Sonazoid is available in Japan, Korea, Norway, Taiwan, Singapore, and China and is expected to be available in the rest of Europe in the near future.

The commercially available UCAs are coated microbubbles composed of gases with a higher molecular weight than air, such as perfluorobutane, perfluorohexane, and sulfur hexafluoride, stabilized by a shell consisting of phospholipid, albumin, or polymer.

\section{Perfluorobutane gas-containing microbubble, Sonazoid}

Sonazoid is composed of microbubbles of perfluorobutane gas coated with hydrogenated egg phosphatidylserine sodium (H-EPSNa). This UCA was approved in 2007 in Japan, in 2012 in Korea, in 2017 in Taiwan, and in 2018 in China for imaging of focal liver lesions (FLLs). As an additional indication, Sonazoid received approval in Japan in 2012 for the imaging of focal breast lesions. After intravenous (IV) injection, vascular distribution of Sonazoid occurs and the agent is taken up by Kupffer cells [2-4]. Therefore, hepatic nodules can be evaluated in the vascular as well as the postvascular (Kupffer) phases with this UCA. Masatoshi Kudo from Japan developed an innovative Sonazoid-based method known as defect reperfusion imaging, which provides improved accuracy for the diagnosis and treatment monitoring of hepatocellular carcinoma (HCC) $[5,6]$.

\section{Contrast-enhanced ultrasound meetings in Asia}

The first international CEUS meeting in Asia, the "International Symposium on Ultrasound Contrast Imaging (ISUCI)," was organized by Professors Fuminori Moriyasu and Shintaro Beppu. The first meeting was held in Kyoto in 1999, coinciding with the approval and launch of Levovist in Japan. Subsequently, annual meetings were held in Tokyo and Osaka alternately until 2008. Sonazoid was approved and launched in Japan in 2007, when the ninth meeting was held in Osaka.

Another international CEUS meeting in Asia, the Asian Congress of Ultrasound Contrast Imaging (ACUCI), was first held in 2009 in Kunming, China, organized by Professor Min Hwa Chen. Since then, the ACUCI meeting has been held annually in different Asian cities. The second ACUCI was held in Tokyo, Japan, in 2010; the third ACUCI in Kunming, China, in 2011; the fourth ACUCI in Seoul, Korea, in 2012; the fifth ACUCI in Taipei, Taiwan, in 2013; the sixth $A C U C I$ in Yokohama, Japan, in 2014; the seventh ACUCI in Guangzhou, China, in 2015; the eighth ACUCl in Kyoto in 2016.

The ninth ACUCI was held in Taipei in 2017, in conjunction with the World Federation for Ultrasound in Medicine and Biology (WFUMB) congress, and the 10th ACUCI in Korea (2018), in conjunction with the Asian Federation of Societies for Ultrasound in Medicine and Biology congress.

\section{Contrast-enhanced ultrasound guidelines}

CEUS has been widely used in Europe since the early 2000s. The European Federation of Societies for Ultrasound in Medicine and Biology (EFSUMB) published its first guideline in 2004. This guideline was revised in 2008, and was propagated to Asia $[7,8]$.

The WFUMB began revising its CEUS guideline in 2010. In February 2013, the WFUMB guideline was published simultaneously in two journals, Ultrasound in Medicine and Biology, the official journal of the WFUMB, and UItraschall in der Medizin, the official journal of the EFSUMB [9-11].

The number of publications in the field of CEUS is increasing rapidly. According to a PubMed search in April 2019, more than 8,400 articles have been published. Among these, the percentages of articles related to the liver and Sonazoid are $24 \%$ and $6 \%$, respectively [12].

\section{Basics}

\section{Contrast imaging}

Contrast imaging is a diagnostic imaging technique for visualizing a region of interest with increased image contrast. Major diagnostic imaging modalities, including X-ray, computed tomography (CT), magnetic resonance imaging (MRI), and US imaging, have a contrast imaging mode. However, the functions of UCAs are different because the principles of the techniques are different.

A high-molecular weight substance such as iodine is used as a contrast agent for X-ray and CT because of its strong X-ray absorption. For MRI, a paramagnetic metal ion such as gadolinium is used to enhance the difference in relaxation times of protons. In diagnostic US, microbubbles of a few microns in diameter are used as a contrast agent. When microbubbles oscillate under US exposure, they generate specific backscattered US signals, in 
addition to the primary US signal. Because the additional US signals from microbubbles have a high amplitude and a specific signature compared to those scattered from tissue, these microbubble agents are referred to as UCA. Imaging of the backscattered US enables an exquisitely detailed visualization of small vasculature containing the contrast agent bubbles with high contrast. Contrast images are still subject to various limitations, such as frame rate, penetration depth, and spatial resolution, which are due to the physical characteristics of US.

\section{Ultrasound Contrast Agents}

A UCA consists of a suspension of bubbles no more than a few microns in diameter. After the UCA is injected IV, the bubbles first pass through the pulmonary capillary bed, after which they spread out into the entire body, including the hepatic circulation. The bubbles must therefore be smaller than the size of the red blood cells, and their resonant size must correspond to US frequencies in the clinical diagnostic range. Each bubble contains a minimally diffusible gas, encapsulated within a coating or shell that prevents diffusion of the gas. This enables the bubbles to remain stable for long enough to allow complete diagnostic imaging. Sonazoid is composed of microbubbles of perfluorobutane encapsulated in a shell of egg phosphatidylserine, allowing them to flow freely in the vascular bed before being taken up by Kupffer cells [2]. The presence of the shell also changes the affinity of the bubbles to surrounding cells. Bubbles phagocytized by Kupffer cells have an extended lifetime of 10 minutes or more, enabling postvascular phase (Kupffer phase) imaging.

\section{Mechanical index as an index of bubble oscillation}

The volumetric change during the bubble expansion under the conditions of negative US pressure is larger than that during bubble contraction under positive US pressure. The volumetric changes also increase with increased duration of the negative pressure. Since larger bubble oscillation causes higher risks for biological tissue, the mechanical index (MI), defined by the following equation, is used as a safety index for mechanical effects of US exposure [13].

$$
M l=p_{\mathrm{r}} / \sqrt{f_{c}},
$$

where $p_{\mathrm{r}}$ is the peak negative pressure of a diagnostic US pulse, and $f_{c}$ is the center frequency of US pulse normalized by $1 \mathrm{MHz}$.

For efficient and safe use of a contrast agent, a standardized index that can quantify bubble oscillation is important. Ml is utilized for this purpose. However, clinical practitioners should be aware that the index is sensitive neither to differences in contrast agent type, nor to spatial inhomogeneity of US pressure.

\section{Signals from oscillating bubbles}

There is a large difference in the characteristic acoustic impedance between contrast agent microbubbles and biological tissue, namely, that irradiated microbubbles scatter US at near-100\% efficiency. Under conditions in which the microbubble's radial change is sufficiently smaller than its resting diameter, the radial change is linearly proportional to the pressure change. This generates US with fundamental frequency components that match those of the irradiating US. Under conditions in which there is a larger diameter change, the radius changes nonlinearly, causing the bubble to generate US with harmonic and subharmonic frequency components that are not originally present in the irradiating US. Under conditions of even higher Mls, stronger bubble oscillation causes collapse and subsequent fragmentation and coalescence of the bubble, resulting in the generation of US containing short-duration wide-band frequency components, including nonlinear ones.

\section{Signals from biological tissue}

Pulsed US that transmits through and is reflected by biological tissue is used to generate diagnostic US images. Reflection itself causes no change in frequency components of the reflected US; however, nonlinear propagation caused by the pressure-dependent speed of sound produces different frequency components, i.e., tissue harmonic components, that are not originally present in the irradiating US [14]. This means that images taken using higher MI US contain more harmonic components.

\section{Separation of bubble signal from tissue signal}

Discrimination of a region containing contrast agent bubbles from other biological tissues is achieved by separating a weak bubble signal from a strong biological tissue signal. Various techniques have been developed to achieve better separation [15-17]. Two basic techniques are (1) frequency filtering based on the difference in frequency components and (2) subtraction of signals captured using two transmitting pulses with and without a phase inversion.

The quality of contrast images is degraded by the presence of harmonic frequency components in the biological tissue signal. Under conditions of exposure to lower MI $(<0.3)$ US, secondgeneration and later-generation UCAs provide a sufficient oscillation and a detectable nonlinear signal, whereas the natural harmonic components originating from biological tissue are negligible. Determination of the optimum acoustic pressure is important because the use of low-intensity US also decreases nonlinear oscillation of the bubbles. Nonlinearity exists not only in acoustic phenomena, such as bubble oscillation and US propagation, but also in signal conditioning of received US signals [18]. Clinical practitioners should be aware of the possibility of artifacts appearing 
in contrast images.

\section{Terminology Imaging phases}

Sonazoid CEUS provides three vascular phases: the arterial phase, the portal venous phase, and the late vascular phase (late phase). In the liver, Sonazoid microbubbles are specifically taken up by Kupffer cells $[2,4]$ leading to the observation of a persistent enhancement during the postvascular phase (Kupffer phase) for $>10$ minutes after Sonazoid injection. There exists some overlap between the vascular phase and the postvascular phase (Kupffer phase) [3]; however, the diagnostic value of the overlap has not yet been well established.

\section{Enhancement features}

Qualitative assessment

The imaging appearance of a region of interest should be described with respect to the degree and distribution of enhancement of each imaging phase and dynamic changes in enhancement features. The degree of enhancement of an observation is described as either hyperenhancing, isoenhancing, or hypoenhancing in comparison to the adjacent normal tissues [9]. Complete absence of enhancement can be described as nonenhancing. For the postvascular phase (Kupffer phase), the term "defect" can be used for nonenhancing or markedly hypoenhancing observations, indicating a deficiency in functioning Kupffer cells [5]. The distribution of enhancement reflects the distribution of microbubbles within the region of interest and enhancement can be reported as either diffuse or focal, either homogeneous or heterogeneous, and either rim-like or nodular. By combining qualitative information from the vascular phases, dynamic enhancement patterns can be described using the following sets of terms:

- Wash-in and wash-out (hyperenhancement in the arterial phase and hypoenhancement in the later phase)

- Sustained enhancement (continuous hyperenhancement or isoenhancement over time), or progressive delayed enhancement (hypoenhancement or isoenhancement in the arterial phase and hyperenhancement in the later phase), based on dynamic changes in the degree of enhancement relative to the adjacent normal parenchyma

- Centripetal (from periphery to center) or centrifugal (from center to periphery) enhancement, based on temporal direction of enhancement within the region of interest.

\section{Quantitative assessment}

Quantitative CEUS parameters reflecting tissue vascularity can be obtained from time-intensity curve (TIC) analysis $[19,20]$. The fitted $\mathrm{TIC}$ of a region of interest can be obtained on a pixel-by-pixel basis using dedicated software. Common parameters from TIC analysis include:

- Peak intensity (PI): maximal signal intensity

- Time to peak (TTP): time from the starting point to the PI of the curve

- Rise time: TTP minus the time at which the maximum slope tangent intersects the $\mathrm{X}$-axis

- Full width of half the maximum: duration between two time points of half of maximum intensity in the TIC

- Wash-in rate: maximum slope tangent at the ascending part of the TIC

- Wash-out rate: maximum slope tangent at the descending part of the curve

- Area under the curves (AUCs) including AUC during washin (WiAUC), AUC during wash-out (WoAUC), and WiAUC plus WoAUC.

\section{Chapter 1: Technical Aspects}

\section{Pharmacokinetics}

Sonazoid consists of microbubbles of perfluorobutane gas encapsulated by an H-EPSNa shell with a mean size of $2.6 \mu \mathrm{m}$. It is administered as an IV bolus injection or as a continuous infusion. After an IV administration, the microbubbles transit through the pulmonary capillaries and reach the left ventricle and then enter to the systemic circulation. For all tissues that include the blood, liver, spleen, fat, kidney, muscle, heart, lung and brain, the $C_{\max }$ value was observed at 5-10 minutes after the injection. Elimination half-life is 30-45 minutes and most is excreted through the lungs [21]. Sonazoid is phagocytosed by Kupffer cells in the liver, which is responsible for postvascular phase images [2-4]. In a healthy volunteer study, the mean arrival times of Sonazoid in the hepatic artery, portal vein, and hepatic vein were 19.2, 24.3, and 32.2 seconds after the injection, respectively [3]. Within 5 minutes after injection, liver parenchymal enhancement reached a plateau and persisted for at least 2 hours [3]. The average time to maximum intensity of the liver parenchyma was about 40 minutes after the injection [3].

\section{Imaging Techniques}

Sonazoid CEUS is obtained with US systems, which are equipped with the same software as that for other second-generation UCAs, such as contrast-specific imaging (contrast imaging) and tissue harmonic imaging (harmonic imaging). When using contrast imaging, parameters such as modulation methods (e.g., phase or amplitude modulation) and MI levels (e.g., low or high MI) are decided depending upon the different imaging techniques of 
Sonazoid CEUS. Harmonic imaging can also be applied to Sonazoid CEUS under low MI.

Different imaging techniques have been developed for Sonazoid CEUS and are characterized by different parameter settings. The most popular technique is low-Ml contrast imaging. It is similar to that for other second-generation UCAs, in that it is conducted with contrast imaging set at a low MI. Two alternative imaging techniques, high-MI contrast imaging and low-Ml harmonic imaging, are considered useful under specific conditions.

\section{Low Mechanical Index Contrast Imaging Scanning method}

With this technique, scanning is conducted at a low MI, using a pulse inversion mode or amplitude modulation mode that changes the amplitude or the phase of the emission phase and receives nonlinear signals during continuous real-time scanning. The level of low $\mathrm{MI}$ in this technique usually ranges from 0.2 to $0.3[10,22-37]$. However, the range of $\mathrm{Ml}$ can be reduced to 0.1 [3,38-41] and can be expanded to 0.4 [3]. Typically, a higher MI level can be used for Sonazoid than for other second-generation UCAs (as low as 0.05) [10], thanks to the specific resistance of Sonazoid microbubbles to the acoustic pressure. The location of the beam focus is set at the posterior margin of the liver and the frame rate is around 10 and up to 15 per second $[22,26,31,42]$.

\section{Imaging acquisition method}

Imaging acquisition during the vascular phase is principally similar to that of other second-generation UCAs, in that the vascular phase includes the arterial, portal venous, and late phases, when microbubbles are confined to the vascular space following IV UCA injection, with slow degradation over 5 minutes [10,26,43-45]. For the arterial phase, the time window for imaging acquisition begins as early as $10-20$ seconds $[10,37,40,45]$, continues to $30-50$ seconds for optimal timing $[10,31,37,38,40,45]$, and is considered to last up to 1 minute after UCA injection $[24,26,28,32,34,38,45]$. Nonlinear signals during this phase reflect the arterial enhancement of an organ or a lesion, providing information on its arterial supply or vascularity. The portal venous phase starts from 30 to 50 seconds $[10,40,42,45]$, continues to $80-90$ seconds for optimal timing $[26,31,37,38]$, and is considered to last until 2 minutes after UCA injection $[10,24-26,37,38,40,42,45]$. The late vascular phase continues after the portal venous phase, and is defined to last until microbubbles disappear from the vascular space $[10,31,38,40,45]$. Depending upon the study design, the portal and/or late vascular phases are not necessarily obtained within the three parts of the vascular phase [22-25,27-29,32-37,41,42,46-48].

Following the late vascular phase, an additional and specific phase is available with Sonazoid CEUS, and this is referred to as the postvascular phase $[2,4,49]$. The postvascular phase follows the late vascular phase, and the corresponding imaging acquisition window is generally considered to begin 10 minutes after the contrast injection when microbubbles are sufficiently accumulated in the Kupffer cells by phagocytosis and are nearly removed from circulation; this phase persists for several hours [22,24$26,28,29,31-34,40-42,45,46,50]$. Most malignant lesions become hypoechoic from the portal phase to the postvascular phase, whereas most benign hepatocyte-origin lesions are iso- or hyperechoic during these phases. These differences are advantageous for characterizing lesions [51-53]. In particular, malignant lesions such as HCC appear as a nonenhancing or hypoenhancing defect in this phase due to intralesion depletion of the Kupffer cells; this property further increases Sonazoid CEUS diagnostic performance, making it comparable with gadolinium ethoxybenzyl diethylenetriamine pentaacetic acid (Gd-EOB-DTPA)-enhanced MRI $[22,51,54,55]$.

\section{Injection method}

Injection methods for the second-generation UCAs are vendorspecific and application-specific, and product package inserts should be consulted. The vendor-recommended dose for Sonazoid CEUS of the liver is $0.015 \mathrm{~mL} / \mathrm{kg}$ (body weight) of the suspension, which is equivalent to $0.12 \mu \mathrm{L} / \mathrm{kg}$ (body weight) of Sonazoid microbubbles, and is prepared by mixing one vial of Sonazoid powder $(16 \mu \mathrm{L})$ and $2 \mathrm{~mL}$ of distilled water $[42,45]$. The suspension is IV administered as a bolus through the antecubital vein, followed by a flush of normal saline or $5 \%$ glucose solution (up to $10 \mathrm{~mL}$ ). Due to the improved sensitivity of recent US systems in detecting microbubbles, injection dose can be reduced to a fixed dose of $0.2-0.7 \mathrm{~mL}$ $[22,24,25,31,32,37,45]$ or to a body weight-based dose of $0.0075-$ $0.010 \mathrm{~mL} / \mathrm{kg}[23,27-30,33-36,40-42,45,47]$.

Defect reperfusion imaging or double contrast US, a sophisticated injection method using Sonazoid, has been introduced for more accurate diagnosis, localization and treatment guidance, and involves reinjection of Sonazoid during the postvascular phase $[5,6,56,57]$. If a hypoechoic defect is seen during the postvascular phase after the first IV injection of Sonazoid, it can be confirmed as HCC if it shows arterial enhancement following the second injection of Sonazoid during the postvascular phase of the first injection $[5,6,56,57]$.

\section{Options for the evaluation of tumor vessels}

With low-MI contrast imaging, detailed observation of tumor vessels is possible only for a short duration of the early arterial phase, as they rapidly become obscured due to simultaneous enhancement of the liver parenchyma. Maximum-intensity projection imaging 
(e.g., Microvascular Flow Imaging) is an imaging technique that combines the flash replenishment sequence obtained by short US exposure of high Ml in a fixed plane that contains a hepatic lesion (to burst the microbubbles in that plane) with the maximum intensity holding sequence obtained by that of low MI in the same plane. This technique is useful for evaluating tumor vessels in detail $[39,58,59]$.

\section{Alternative Imaging Techniques \\ High-mechanical index contrast imaging}

Compared to the low-MI contrast imaging technique, high -MI contrast imaging is more sensitive for detecting Sonazoid microbubbles [60] and therefore is better for evaluating tumor vessels and enhancement during the postvascular phase $[61,62]$. Low-MI contrast imaging may give rise to difficulty in differentiating necrotic areas from viable areas of HCCs during the postvascular phase, because both areas may appear as defects, even though the viable areas contain tumor vessels [61]. As with stimulated acoustic emission imaging in the late vascular phase of parenchymal enhancement of the liver with SH U 508A (Levovist, Schering $A G$, Berlin, Germany) [63], when using high-MI contrast imaging, recirculation of Sonazoid microbubbles gushing into tumor vessels of the viable area can be detected better after Sonazoid microbubbles within and around the tumor are destroyed during intermittent scanning, whereas Sonazoid microbubbles will not be seen in the necrotic areas $[61,64]$. To easily destroy Sonazoid microbubbles within and around a tumor, intermittent scanning at 2 frames per second under high $\mathrm{MI}(0.7-1.2)$ is recommended with a reduced dose of $0.2 \mathrm{~mL}[37,61,62]$.

Hyperechoic lesions may not appear as a defect in the postvascular phase under the low-MI contrast imaging mode, compared with background signals. However, high-MI contrast imaging is more sensitive than low-Ml contrast imaging for detecting hypervascular HCCs as a defect in patients with hyperechoic lesions during the postvascular phase $[37,65]$. Deep-seated lesions around $10-12 \mathrm{~cm}$ or over $10 \mathrm{~cm}$ from the skin surface may be poorly observed under low-MI contrast imaging due to US attenuation. High-MI contrast imaging is therefore useful for evaluating tumor vascularity or identifying defects corresponding to deep-seated lesions $[37,66]$.

\section{Three-dimensional contrast-enhanced ultrasound}

High-MI contrast imaging prolongs the observation time for tumor vessels, and enables clear tumor enhancement by eliminating microbubbles in the microvessels. This capability allows automatic acquisition of three-dimensional CEUS of hepatic lesions with a high MI (0.5-0.9) of 8-13 frames per second [67-72]. Tomographic US images and sonographic angiograms are useful for threedimensional vascular characterization and differential diagnosis of hepatic tumors with improved diagnostic performance [67-70]. This imaging technique can also be useful for evaluating the effects of locoregional therapy for HCCs by offering unique three-dimensional visualization $[71,72]$.

\section{Low-mechanical index harmonic imaging}

Low-Ml harmonic imaging is obtained using conventional gray-scale US images combined with a newly developed transducer, which consists of wide-band phase inversion harmonic gray-scale imaging, and provides a high spatial resolution and deep penetration with high frame rates of 24-30 frames per second [48,73-75]. Using this transducer, once the target lesion is detected, the $\mathrm{MI}$ can be reduced to around $0.18-0.28$ manually. Subsequently, Sonazoid injection can provide a detailed evaluation of tumor vessels and tumor staining in real time, owing to the high frame rate.

\section{CQ1: Is defect reperfusion imaging useful?}

Consensus Statement \#1

Defect reperfusion imaging is useful for accurate diagnosis, localization and treatment guidance of HCC. (A-1)

(Agreement level 9.1, evidence level 7.8, recommendation level 8.7)

\section{CQ2: Is high MI-contrast imaging useful?}

Consensus Statement \#2

It can be useful for differentiating necrotic areas from the viable areas of HCC and evaluating hyperechoic lesions and deep-seated lesions. (B-1)

(Agreement level 8.1, evidence level 7.0, recommendation level 7.9)

\section{Chapter 2: Detection of Focal Liver Lesions}

\section{Focal Hepatic Lesions}

Detection of FLLs is the initial step in the management of HCC. Imaging diagnosis of HCC is usually based on the observation of hemodynamic hallmark features from dynamic vascular phase imaging with nonorgan-specific extracellular contrast agents, regardless of imaging modality. In the 21st century, various liverspecific contrast agents have been used in clinical practice and have expanded the range of diagnosis. Sonazoid, a liver-specific contrast agent for US, allows imaging in the postvascular phase (Kupffer phase) as well as the dynamic vascular phases. This postvascular phase (Kupffer phase) contrast enhancement provides a stable time window of $>60$ minutes, which allows enough time for the examination of the entire liver. In contrast to other UCAs, Sonazoid has a unique potential to be used for the detection of HCC in various settings, including disease surveillance.

Kudo et al. [6] reported results from a prospective study using 
Sonazoid CEUS with a reinjection technique in HCC surveillance. In 292 patients under HCC surveillance, they found 16 additional HCCS on CEUS that were not detected on B-mode US. A prospective randomized controlled trial conducted in Japan clearly showed the ability of Sonazoid CEUS to depict significantly smaller lesions (mean \pm standard deviation [SD], $13.0 \pm 4.1 \mathrm{~mm}$ ) than B-mode US $($ mean $\pm S D, 16.7 \pm 4.1 \mathrm{~mm})(P=0.011)$ [76]. However, the costbenefit balance and survival benefit of Sonazoid CEUS for the early detection of $\mathrm{HCC}$ are still controversial.

In a liver segment-based retrospective analysis using dynamic CT as a reference standard, Goto et al. [30] reported rather mediocre detection sensitivity of Sonazoid CEUS compared with B-mode US; $0.732(90 / 123)$ and $0.831(93 / 123)$ versus $0.837(103 / 123)$ and 0.846 (104/123), respectively. However, the specificity of Sonazoid CEUS was significantly higher than that of B-mode US, 0.982 $(272 / 277)$ and $0.978(271 / 277)$ versus 0.892 (247/277) and 0.949 (263/277). Similar trends were observed in prospectively collected data from five tertiary centers in Korea $[77,78]$. The detection rate of early-stage HCC of Sonazoid CEUS was 1.0\% (95\% confidence interval [CI], $0.3 \%$ to $2.2 \%$; 5 of 524 patients) and that of $\mathrm{B}$-mode US was $0.6 \%(95 \% \mathrm{Cl}, 0.1 \%$ to $1.7 \% ; 3$ of 524 patients), without a statistically significant difference $(P=0.16)$. However, the false referral rate with Sonazoid CEUS was $1.3 \%(95 \% \mathrm{Cl}, 0.5 \%$ to $2.7 \% ; 7$ of 524 patients) and that with B-mode US was $4.6 \%$ (95\% $\mathrm{Cl}, 3.0 \%$ to $6.7 \% ; 24$ of 524 patients), which was statistically significant $(\mathrm{P}<0.001)$. Sonazoid $\mathrm{CEUS}$ did not improve the detection rate of early-stage HCC but did reduce the false referral rate for HCC in patients under HCC surveillance [78].

The results for higher specificity or lower false referral rate with Sonazoid CEUS are consistent across all the studies that have been conducted to date. Considering that a substantial proportion of patients with cirrhosis experience physical harm for false-positive or indeterminate surveillance tests, efforts should be made to reduce false referrals $[79,80]$. There are many regenerative or dysplastic nodules (DNs) in the cirrhotic liver tissue, resulting in a high rate of false-positive diagnosis using B-mode US. Kupffer cells are usually preserved in these nodules, which makes a clear case for the benefit of Sonazoid CEUS in reducing false referral, compared with B-mode US [55].

CQ3: Can surveillance by Sonazoid CEUS be used in the detection of small HCCs in cirrhotic patients?

Consensus Statement \#3

Sonazoid CEUS with postvascular phase (Kupffer phase) can be used in the detection of small HCC (not early HCC) in HCC surveillance, especially in patients with very coarse liver parenchyma. (B-2)

(Agreement level 8.0, evidence level 7.3, recommendation level 7.4)
CQ4: Is Sonazoid CEUS useful in the detection of FLLS, which are not detected by $B$-mode US?

Consensus Statement \#4

Sonazoid CEUS is useful to detect FLLs including HCC or metastasis. $(\mathrm{A}-1)$

(Agreement level 8.9, evidence level 9.4, recommendation level 8.9)

\section{CQ5: What is the effect of adding Sonazoid-enhanced US to $B$-mode US in patients under HCC surveillance?}

Consensus Statement \#5

Addition of Sonazoid-enhanced US to B-mode US can reduce false referrals for dynamic $\mathrm{CT}$ or MRI in patients under HCC surveillance. (B-2)

(Agreement level 7.8, evidence level 7.1, recommendation level 7.3)

\section{Intraoperative Ultrasound \\ Background}

In intraoperative US (IOUS) with laparotomy, the US probe can be placed directly on the liver capsule, via the laparotomy incision, avoiding interference from surrounding skin, fat, and muscle tissue. IOUS is therefore more sensitive for detecting FLLs than transabdominal US [81]. Furthermore, IOUS may facilitate liver parenchymal transection by allowing surgeons to visualize the relationships among the tumors, intrahepatic vessels, and transection lines [82]. IOUS thus has become an essential tool for liver surgery. The advent of UCAs has enabled contrast-enhanced IOUS (CE-IOUS), which adds to the utility of intraoperative use of US.

\section{Instruments for contrast-enhanced intraoperative ultrasound}

A dedicated US transducer for the intraoperative setting, which is small enough to be handled with two fingers, is used for both noncontrast IOUS and CE-IOUS. Both linear and convex transducers can be used; the former provides somewhat superior contrast and spatial resolution, whereas the latter can provide a wider field of view and is easier to handle. In general, the IOUS machine and ultrasonography mode settings are similar to those used for transabdominal US.

\section{Contrast-enhanced intraoperative ultrasound technique}

Following a laparotomy and minimal liver mobilization, conventional B-mode IOUS is performed to confirm tumor staging with reference to preoperative images. Sometimes, new FLLs that have not been identified in preoperative imaging are found by IOUS, and subsequently, CE-IOUS is useful for characterizing these FLLS. A peripheral venous injection of $0.5 \mathrm{~mL}$ of Sonazoid mixture (commercially recommended ratio) is usually selected. The detailed 
methods of vascular and postvascular phase (Kupffer phase) imaging are almost the same as for transabdominal CEUS. During the waiting time between the vascular phase and the postvascular phase (Kupffer phase), usually around 10-15 minutes, additional liver mobilization or cholecystectomy can be performed; however, division of hepatic arteries or portal veins should be avoided to obtain full contrast effects in the liver. As with transabdominal CEUS, defect reperfusion imaging is useful in CE-IOUS for further characterization of the FLLS.

\section{Image interpretation}

Characterization of the FLLs is the same as that for extracorporeal CEUS.

\section{Recommended use}

First, vascular phase and postvascular phase (Kupffer phase) CEIOUS may be used for final characterization for FLLs that were either indeterminate at preoperative imaging or newly identified during nonenhanced IOUS $[83,84]$.

Second, whole-liver screening during the postvascular phase (Kupffer phase) enables the identification of a new tumor as a contrast defect; this has been reported in around $10 \%-20 \%$ of all patients undergoing $\mathrm{HCC}$ resection [84] or colorectal liver metastasis resection [85]. Even very small tumors with $2 \mathrm{~mm}$ in diameter can be identified with CE-IOUS despite not being detected at preoperative imaging with Gd-EOB-DTPA-enhanced MRI and/or IOUS. During surgery for HCC, the vascularity of such new FLLs identified during the postvascular phase (Kupffer phase) can be evaluated using the defect reperfusion technique.

Third, postvascular phase (Kupffer phase) CE-IOUS may be able to detect and facilitate resection of disappearing tumors of colorectal liver metastases after effective chemotherapy that are missed at preoperative imaging [86].

Fourth, CE-IOUS may be used as a navigation tool during the liver parenchymal transection; surgeons can obtain clear images of tumors as defects and can recognize the relationships among the transection line, major vessels, and the tumor simultaneously. This utility of CE-IOUS is most apparent when a tumor is poorly visualized by nonenhanced IOUS. Because the contrast effect of the postvascular phase (Kupffer phase) lasts for over an hour, sometimes several hours, CE-IOUS can perform this role during the entire resection procedure. Attenuation of contrast effect can be overcome by an additional injection of Sonazoid.

\section{Supported use in anatomical hepatic resection}

Sonazoid can be injected into the intrahepatic portal venous branch to estimate the volume which is fed by the target portal branch. Surgeons can confirm that injected microbubble is correctly perfused into the targeted segments and thereby can determine the transection lines by following the border of the segments of the liver surface $[87,88]$. This technique is useful for accurate anatomical hepatic resection. Furthermore, surgeons can determine whether the tumor is completely contained within their proposed anatomical hepatic resection.

\section{CQ6: What is the advantage of the use of Sonazoid CE- IOUS?}

Consensus Statement \#6

CE-IOUS can clearly depict liver tumors and identify new tumors that have not been detected at preoperative imaging. (A-1)

(Agreement level 9.0, evidence level 7.9, recommendation level 8.9)

\section{Chapter 3: Characterization of Focal Liver Lesions}

\section{In Noncirrhotic Liver \\ Background}

Because Sonazoid microbubbles are taken up in Kupffer cells in the hepatic sinusoid $[2,4]$, normal parenchyma or focal hepatic lesions that contain Kupffer cells will present with bright enhancement on the postvascular (Kupffer phase) imaging (10 minutes or later). Conversely, hepatic tumors in which tumor cells replace both Kupffer cells and hepatic sinusoid cells show enhancement defects in the postvascular phase imaging. Therefore, the interpretation of findings in the postvascular phase imaging in patients without cirrhosis is dependent on the presence of Kupffer cells in focal hepatic disease.

\section{Benign hepatic diseases}

Hemangioma

Like other UCAs, Sonazoid exhibits peripheral rim enhancement $(45 \%)$ and peripheral nodular enhancement (43\%) patterns in hemangiomas and filling in a centripetal direction in the vascular phase. In an observational study, classification (diagnostic) accuracy for hemangioma was $93.3 \%$ [89]. In addition, postvascular phase imaging exhibits isoenhancement or slight hypoenhancement relative to surrounding liver parenchyma $[44,90]$. In a case report on diagnosis of sclerosed hemangioma, postvascular phase imaging showed a defective lesion similar to hepatic malignancy, such as intrahepatic cholangiocarcinoma (ICC) and metastasis; the findings indicated that reinjection of a contrast agent could help discriminate such contrast defects from malignancy as hyalinized tissue was not enhanced [91].

Focal nodular hyperplasia

Focal nodular hyperplasia (FNH) is a benign hepatic lesion that 
is often an incidental finding. In a study comparing Kupffer cell numbers in different types of hepatic tumors, the number of Kupffer cells in FNH was significantly greater on average than that in HCC, especially in HCC with moderate or poor cellular differentiation [51]. In Sonazoid CEUS for the evaluation of FNH, 24 of 31 lesions (77.4\%) displayed pathognomonic imaging patterns such as central artery and stellate vascularity. In most cases (93.5\%), postvascular phase imaging revealed a isoechoic or slightly hyperechoic appearance compared with the surrounding liver parenchyma [26].

\section{Adenoma}

Hepatocellular adenoma (HCA) is a benign tumor which frequently bleeds and rarely transforms into HCC. Histologically, there are four subtypes of HCA: inflammatory; hepatocyte nuclear factor (HNF) 1a -inactivated; Wnt/ $\beta$-catenin-associated; and unclassified subtype, from most to least common [92]. In a CEUS study using SonoVue [93], HNF1a-inactivated HCA showed isovascularity or moderate hypervascularity in the arterial phase and isoenhancement in the portal venous phase. In contrast, inflammatory HCA showed features of centripetal filling, linear vascularity, peripheral rim-like sustained enhancement, and central wash-out in the late vascular phase. Unfortunately, there are no Sonazoid CEUS studies on imaging findings in HCA, except for a case report on inflammatory HCA [94]. In this report, Sonazoid CEUS showed mild global hyperenhancement with inflow of microbubbles from the tumor periphery in the arterial phase, persistent enhancement in the portal venous phase, and mild heterogeneous hypoenhancement in the postvascular phase.

Focal fatty change

Focal fatty change, either fat infiltration or fatty sparing, may appear as masses on baseline US. Differential diagnosis is important, especially in patients with underlying malignant disease or with an atypical location of suspected focal fatty changes. Sonazoid CEUS shows isoenhancement throughout all phases [20].

Liver abscess

Liver abscesses show varied appearance on the baseline US. In contrast, most liver abscesses show peripheral enhancement in the vascular phase and unenhanced central areas in the postvascular phase. The appearance of liver abscesses is clearer on Sonazoid CEUS than on conventional US [95].

Other benign lesions

Cysts show no contrast enhancement at all. Sonazoid CEUS is not necessary for simple cysts but is useful to evaluate complicated or atypical cysts. If only postvascular phase images are available, cysts can mimic malignant liver lesions by showing discrete defects [20].

Hepatic angiomyolipoma ( $A M L$ ) is a rare benign mesenchymal tumor with heterogeneous echogenicity on baseline US. Sonazoid CEUS shows arterial hyperenhancement and contrast defect in the postvascular phase, falsely suggesting malignancy [96]. In the case of fat-containing AML which shows strong hyperechogenicity on baseline US, it might be difficult to observe the contrast defect in the postvascular phase.

Inflammatory pseudotumor is a rare disease whose definitive diagnosis is usually made at surgery and biopsy. Sonazoid CEUS shows isoenhancement in the early vascular phase and hypoenhancement in the postvascular phase, which is difficult to distinguish from malignant tumors [97].

\section{Malignant hepatic diseases}

Intrahepatic cholangiocarcinoma

ICC is relatively rare and comprises $3 \%-7 \%$ of primary liver cancers. The Liver Cancer Study Group of Japan has proposed a classification of ICC based on macroscopic features: mass-forming, periductal infiltrating, and intraductal, or mixed mass-forming, and periductal infiltrating [98]. Approximately $80 \%$ of ICCS are classified as the mass-forming type.

The major characteristics of ICC on B-mode US are dilated distal bile duct and irregular tumor border. The enhancement patterns of ICC on Sonazoid CEUS are rim-like enhancement in the early arterial phase followed by complete defect in the postvascular phase $[48,99,100]$. ICC can show inhomogeneous enhancement during the arterial phase, such as rim-like enhancement (44.4\%), inhomogeneous enhancement (11.1\%), or inhomogeneous hypoenhancement (44.4\%) [101]. These dynamic findings are mostly overlapped with those of liver metastases.

\section{Metastasis}

Since most metastatic lesions that do not contain Kupffer cells appear as clear lesion defects, in contrast to the background of normal parenchyma that shows hyperenhancement on the postvascular phase, workup testing for hepatic metastasis is a reasonable indication for Sonazoid CEUS $[48,62]$. The diagnostic performance of Sonazoid CEUS is higher than that of B-mode US $[90,102]$ and comparable to that of contrast-enhanced CT (CECT) [103]; however, it is not better than those of superparamagnetic iron oxide (SPIO)-enhanced MRI and Gd-EOB-DTPA-enhanced MRI [103]. Like an ICC, rim-like arterial phase hyperenhancement appears in the peripheral portions of the tumor, and wash-out follows it earlier than that of HCC [104]. In the postvascular phase, metastatic lesions are clearly demarcated and more hypoenhanced, compared with benign lesions. 
Lymphoma

Lymphoma shows homogeneous hypoechogenicity on baseline US. On Sonazoid CEUS, lymphomas show early homogenous hyperenhancement and early wash-out in the vascular phase and defect in the postvascular phase [105].

CQ7: Can Sonazoid CEUS diagnose common benign focal lesions in patients with noncirrhotic liver?

Consensus Statement \#7

Sonazoid CEUS enables diagnosis of common benign focal lesions such as hemangioma, FNH or focal fatty deposit. (B-1)

(Agreement level 8.6, evidence level 8.1, recommendation level 8.9)

\section{CQ8: Can Sonazoid CEUS diagnose malignant focal lesions in patients with noncirrhotic liver?}

Consensus Statement \#8

Sonazoid CEUS enables diagnosis of malignant focal lesions such as ICC and metastasis. (B-1)

(Agreement level 8.2, evidence level 7.7, recommendation level 8.5)

\section{In Cirrhotic Liver}

\section{Background}

Risk factors for primary liver cancers such as HCC or ICC include hepatitis B or hepatitis C infection, cirrhosis, nonalcoholic steatohepatitis, and diabetes mellitus [106]. Annual incidence rates of HCC show an increasing trend in patients with developing cirrhosis [107]. Therefore, US surveillance of patients with chronic liver disease is essential (1) to improve early tumor detection and diagnosis of liver cancers, and (2) to provide an opportunity for curative treatment.

Sonazoid contrast-enhanced ultrasound imaging for cirrhotic patients

US imaging investigation starts with conventional B-mode and Doppler techniques, and the characteristics of B-mode images and dynamics of lesion enhancement can be used to diagnose FLLs [108]. Interpretation of dynamic enhancement imaging is based on the patterns observed in the vascular and postvascular phases [108]. Hepatic malignancies, including HCCs, are mostly visualized as defects in the postvascular phase. An additional contrast agent injection is performed to confirm tumor vessel flow in the defect, a technique known as defect reperfusion imaging [109]. Defect reperfusion US imaging can be helpful to detect malignant nodules that are missed on B-mode US, such as small HCC or recurrent HCC. However, the liver parenchyma often has a coarse appearance of being diffusely atrophied on US in patients with severe cirrhosis. Depletion and/or impaired function of the Kupffer cells are often observed in patients with severe liver cirrhosis. Therefore, detection and differentiation of benign or malignant FLLs on a cirrhotic background can be a challenge.

\section{Hepatocarcinogenesis}

The malignant transformation of hepatocytes to HCC is a multistep process associated with genetic mutations, allelic losses, epigenetic alterations, and perturbation of molecular cellular pathways $[110,111]$. The phenotypic expression of these changes accompanies HCC development spatially and temporally, and can manifest as precursor lesions, termed DNs. In the course of hepatocarcinogenesis, these nodules show a malignant transformation to HCC with a shift in vascular supply from the portal vein to the hepatic artery and an increase in size.

In the early stages of hepatocarcinogenesis, such as DNs and early HCCs, hepatocytes show increased fat accumulation compared to that of the background liver [112]. The incidence of fat accumulation is increased in DNs and early HCC. The mechanism of fat accumulation in early hepatocarcinogenesis is presumed to be due to the deficient development of unpaired arteries and reduction of portal venous and nontumoral arterial flow, which induces intracellular fat accumulation in an ischemic/hypoxic environment [113].

\section{Characterization of focal liver lesions}

Hepatocellular carcinoma

The characteristics of classical HCC on B-mode US are a mosaic pattern, septum formation, peripheral sonolucency (halo), and perinodular daughter nodule formation [108]. Color Doppler imaging demonstrates arterial pulsating flows, such as basket pattern flow and "spot" pattern flow; these patterns reflect the presence of a fine network of arterial vessels surrounding the tumor nodules [108].

The typical dynamic enhancement patterns of HCC are hyperenhancement in the arterial phase, followed by iso/ hypoenhancement in the portal phase and defects in the postvascular phase (Kupffer phase) $[10,108]$. In more than $97 \%$ of cases, this pattern corresponds to HCC $[48,99]$. Arterial hyperenhancement usually appears homogeneous but may be heterogeneous in larger nodules $(>3 \mathrm{~cm})$, which contain regions of necrosis. Rim enhancement is atypical for HCC.

Early hepatocellular carcinoma

Early HCC lesions can be composed mainly of well-differentiated $\mathrm{HCC}$, and nonexpansive growth of early HCC can often result in a poorly demarcated margin. Early HCC lesions often exhibit fatty changes, and diffuse fat accumulation is manifested in $40 \%$ of early 
HCCs. Major US characteristics of early HCC are as follows: homo-I heterogeneous hyperechoic mass lesions and nodule-in-nodule appearance $[48,99,100,114,115]$. A nodule-in-nodule appears as a hypoechoic spot within a hyperechoic mass lesion. On US screening for HCC, these features are often seen in HCC nodules that measure $11-20 \mathrm{~mm}$ in diameter.

On Sonazoid CEUS, arterial hypovascularity with portal blood supply is generally present in early HCC. In cases with a nodulein-nodule appearance, hyperenhanced HCC nodules indicate welldifferentiated HCC with fatty changes, whereas inner hypoenhanced spots indicate moderately differentiated HCC without fatty changes. Therefore, hypervascular foci appear within a hypovascular nodule.

Kupffer cells can remain inside DNs and early HCC lesions, which contain blood spaces more similar to normal sinusoids. The differentiation between a DN and early HCC is difficult because neither show clear defects in the postvascular phase (Kupffer phase). However, hypovascular nodules that are hypoenhanced in the postvascular phase (Kupffer phase) may be diagnosed as early HCCS [116].

\section{Dysplastic nodule}

DNs show inhomogeneous and reticular patterns of hypervascularity in the early arterial phase, followed by isoenhancing or slightly hypoenhancing appearance in the portal phase to the postvascular phase (Kupffer phase) $[48,99,100,114-116]$. However, differentiating between high-grade DN and early HCC with imaging is challenging, even when using Sonazoid CEUS.

Regenerative nodule

Regenerating nodules show iso/hypovascular enhancement in the vascular phase with no apparent Kupffer cell defect $[48,99,100,114-116]$.

\section{Monitoring changes in enhancement patterns}

An inconclusive CEUS pattern does not rule out malignancy and should prompt dynamic CT or MRI. If these are also inconclusive, a biopsy is needed. If this is negative, the nodule should be followed up every 3-4 months, and if it enlarges or the enhancement pattern changes, diagnostic investigations should be resumed [117].

CQ9: Is CEUS useful to evaluate nodules during hepatocarcinogenesis such as DN, early HCC, or HCC?

Consensus Statement \#9-1

CEUS is useful to evaluate overt HCC. (A-1)

(Agreement level 9.5, evidence level 9.1, recommendation level 9.2) Consensus Statement \#9-2

CEUS can be useful for characterization of DN and early HCC. (B-1)
(Agreement level 8.1, evidence level 7.6, recommendation level 7.9)

CQ10: Is CEUS useful to diagnose non-HCC malignancies including ICC or metastasis in patient with cirrhotic liver?

Consensus Statement \#10

CEUS is useful to diagnose non-HCC malignancies including ICC or metastasis. (B-1)

(Agreement level 8.5, evidence level 7.5, recommendation level 8.3)

\section{Portal Vein Thrombosis}

Portal vein thrombosis (PVT) is a common complication of portal hypertension, characterized by a complete or partial obstruction of the portal blood flow [118]. The prevalence of PVT is approximately $1 \%$ in the general population at necropsy and $0.6 \%-26 \%$ in patients with cirrhosis [118]. The presence of PVT may be associated with the worsening of liver function and severity of portal hypertension. Portal vein tumor thrombus is a separate entity and should be clearly differentiated from bland PVT. The typical pattern reported is that of retrograde intrathrombus arterial enhancement $[119,120]$. Diagnostic accuracy with SonoVue for differentiating between bland and malignant PVT is over $90 \%, 92.5 \%$ by Tarantino et al. [121] and $93.3 \%$ by Song et al. [122]. Similarly, for differentiating bland from malignant PVT in patients with HCC, Definity imaging shows 100\% sensitivity and 100\% negative predictive value [123]. However, there is no enough evidence with Sonazoid for this application. Recent studies suggested that Sonazoid may help select potential candidates for anticoagulation in patients suffering from PVT by supporting prediction of therapeutic effect $[124,125]$. As for prediction of the outcome of anticoagulation, positive contrast enhancement with Sonazoid in the PVT suggests successful recanalization [124]. This enhancement is observed at the portal venous phase, but not at the arterial phase.

\section{Macroscopic Classification \\ Background}

Nodular HCC can be subclassified as "simple nodular type (SN)," "simple nodular type with extranodular growth (SNEG)," and "confluent multinodular type (CM)" [126]. The risk of developing malignancy increases with stepwise progression from $\mathrm{SN}$ to $\mathrm{CM}$, correlating with the changes in pathological features, from moderate to poor differentiation. Non-SN is a factor indicating poor prognosis in patients with HCC $[127,128]$. Assessment of macroscopic type provides valuable information for the management of HCC.

\section{Technical aspects and diagnostic features}

HCCs are visualized as defects in the postvascular phase (Kupffer phase), and the shape of the defect can be evaluated with whole- 
tumor scanning. SN-, SNEG-, or CM-type HCCs can be defined as tumors showing a clear round shape, one or more perinodular tumor growths or a cluster of small and confluent nodules, respectively.

Sonazoid CEUS can provide high-quality imaging assessment for determining the macroscopic classification of small nodular HCCS and is more reliable for this evaluation than CECT $[42,129,130]$. The diagnostic capability of Sonazoid CEUS in the postvascular phase (Kupffer phase) is similar to that of MRI with an SPIO [47] or GdEOB-DTPA [131]. However, the diagnosis of macroscopic findings with individual modalities has limitations. Combined diagnosis with CEUS and Gd-EOB-DTPA-enhanced MRI may provide high diagnostic ability.

\section{CQ11: Is Sonazoid CEUS useful for the prediction of macroscopic classification of HCC?}

Consensus Statement \#11

Sonazoid CEUS is useful to predict macroscopic classification of HCC. (B-1)

(Agreement level 7.8, evidence level 7.0, recommendation level 7.4)

\section{Chapter 4: Guiding Intervention}

\section{Biopsy}

When performing percutaneous biopsy for focal hepatic lesions, US has several advantages over CT and MRI. These include real-time imaging, multiplanar imaging, no ionizing radiation, portability, fast and inexpensive examination, and the ability to visualize critical structures such as large blood vessels $[132,133]$. However, one of the main challenges during US-guided biopsy is poor lesion conspicuity on conventional B-mode US [134]. When biopsy targets are not well depicted or not visualized on conventional B-mode US, the use of Sonazoid can be helpful to enhance the visualization of targets $[53,135,136]$. Lesion detection rates were significantly improved during the vascular phase $(84.0 \%)$ and postvascular phase (Kupffer phase) (92.3\%) in comparison to conventional B-mode US (77.3\%) [136].

One study demonstrated that the technical success rate of Sonazoid CEUS (92.3\%) was significantly higher than that of conventional B-mode US (76.8\%) [53]. To the best of our knowledge, this is the only study to compare the technical success rate of biopsy between Sonazoid CEUS and conventional B-mode US. One limitation of this study was that the choice of B-mode US and Sonazoid CEUS was based on the operator's decision, and not based on the predetermined criteria. The reported technical success rates on Sonazoid CEUS ranges from $87.6 \%$ to $92.3 \%$; these numbers are quite high considering that biopsy cannot be even attempted without the use of Sonazoid $[53,135,136]$.
Fusion imaging can also help address issues with poor visualization using conventional B-mode US $[137,138]$. The combination of fusion imaging and CEUS can be a powerful tool to improve lesion conspicuity and technical feasibility of liver biopsy [135]. In addition to better lesion conspicuity, Sonazoid CEUS is beneficial in terms of long-lasting visibility during the postvascular phase (Kupffer phase). The unique postvascular phase (Kupffer phase) imaging capabilities available only with Sonazoid open a longer time window that is sufficient to properly select the route and precisely perform a biopsy. This is in contrast to the limited time window during the vascular phase imaging with other intravascular UCAs, although there is a paucity of data comparing Sonazoid to other intravascular UCAs. CEUS can be helpful to improve the quality of biopsy specimens by avoiding necrotic areas [139]. This is because vascular phase images enable operators to differentiate vascularized areas of targets from necrotic areas $[139,140]$.

\section{CQ12: Is Sonazoid CEUS helpful to perform liver biopsy? \\ Consensus Statement \#12}

Sonazoid CEUS improves target lesion visibility and the technical success rate of biopsy when biopsy targets are poorly visualized in conventional B-mode US. (A-1)

(Agreement level 8.9, evidence level 7.4, recommendation level 9.1)

\section{Interventional Tumor Ablation \\ Background}

Recent advances in imaging techniques such as Gd-EOB-DTPAenhanced MRI and diffusion-weighted imaging allow detection of very small HCCs $[141,142]$. Earlier detection of tumors may lead to better treatment outcomes after tumor ablation, as tumor size is an important factor affecting the therapeutic efficacy. However, localizing such small tumors with conventional B-mode US can be challenging as smaller tumors have a tendency to have poor lesion conspicuity on US [143]. According to previous studies [144,145], $5.2 \%-25.3 \%$ of small HCCs had poor conspicuity on planning US for radiofrequency ablation (RFA). As expected, mistargeting (ablating a pseudolesion rather than a true lesion) can occur after conventional US-guided RFA $[142,146]$. In previous studies, the most common cause of mistargeting was confusion with cirrhotic nodules, followed by a poor conspicuity of the HCC, a poor sonic window, a poor electrode path, and an inaccurate electrode placement. These results are not surprising because hepatitis $B$ virus-related liver cirrhosis is characterized by macronodular cirrhosis in which numerous pseudolesions can mimic true small HCCS on US $[142,146]$. Therefore, CEUS has been widely utilized to accurately localize small target lesions and to guide tumor ablation. 


\section{Guidance of Tumor Ablation}

Many studies reported that Sonazoid CEUS-guided RFA has several advantages over conventional B-mode US-guided procedures $[5,45,49,50,57]$. First, Sonazoid CEUS increases the detectability of small HCCS, compared with B-mode US. In one large study $(n=716)$, the detectability of HCC using Sonazoid CEUS was much higher than that with B-mode US (93.2\% vs. 83.5\%, P=0.04) [50]. A recent study also reported that equivocally conspicuous HCCs surrounded by cirrhosis-related pseudolesions can be accurately localized in up to $72 \%(31 / 43)$ of HCCs using the postvascular phase of Sonazoid CEUS [147]. According to the study, the postvascular phase was especially helpful for identifying tumors with hypo- or isoechoic lesions on the B-mode US. Defect reperfusion imaging is also useful for localizing viable HCCs that have poor lesion conspicuity on the B-mode US. Either locally recurrent HCCs or new HCCs undetectable on the B-mode US can benefit from defect reperfusion imaging with Sonazoid CEUS $[5,148,149]$. Not all small tumors can be localized with CEUS. For example, tumors located in the deep portions of the liver far from the skin may not be identified due to US attenuation on conventional CEUS in which low MI is used. Numata et al. [66] suggested that high $\mathrm{Ml}$ can be applied intermittently during Sonazoid CEUS for localizing these tumors as it can destroy microbubbles in and around the tumor and tumor vessels, and tumoral enhancement may be visible because of backflow into the tumor.

Second, Sonazoid CEUS facilitates accurate applicator placement in the tumor during local ablation therapy. In general, applicator insertion into the tumor can be accurately performed when the target lesion has good conspicuity on the B-mode US. However, when the target lesion does not have good conspicuity on the B-mode US, the postvascular phase is utilized for applicator placement as it has a long temporal window and the target lesion can be seen as a defect $[57,150]$.

Third, Sonazoid CEUS can decrease the number of sessions needed for RFA of HCCs $[50,57,145]$. According to a previous study [50], Sonazoid CEUS guidance reduced the number of RFA sessions compared with B-mode US guidance in a historical cohort (mean $\pm S D$, $1.33 \pm 0.45$ vs. $1.49 \pm 0.76 ; P=0.0019$ ). Sonazoid CEUS is also effective for guiding ablation for HCC with local tumor progression (LTP) that is not clearly identifiable with B-mode US [151]. In the study, the number of treatment sessions was significantly lower in the CEUS group than in the conventional US group (mean \pm SD, $1.1 \pm 0.2$ vs. $1.4 \pm 0.6 ; P=0.037$ ). This was because the technical success rate after a single RFA session was significantly higher in the CEUS group than in the B-mode US group (94.7\% vs. $65.0 \%$, $P=0.043$ ) [151]. Eventually, Sonazoid CEUS guidance can lead to better sustained local tumor control compared to B-mode US guidance. According to a previous study [152], sustained local control rate was much higher in the CEUS-guided RFA group than in the B-mode US-guided group (85.3\% vs. $66.4 \%$ at 2 years).

Fourth, periprocedural assessment of treatment response can be performed with CEUS as it allows evaluation of the extent of the ablation zone at the end of the ablation procedure $[45,153,154]$. $A$ residual viable tumor can be detected during the RFA session using CEUS. In addition, CEUS may allow visualization of immediate complications after RFA such as active bleeding, hemobilia, or segmental infarction of the liver [155]. For these reasons, CEUS may have the potential to reduce the number of CT examinations after RFA [156]. However, a waiting time after thermal ablation is required; this is to allow the echogenic zone to fade out before performing CEUS. Currently, solid data are lacking to support definitive recommendations regarding the waiting time after RFA for CEUS evaluation of immediate post-treatment response. Different waiting times -3 hours [154] or 1 day $[156,157]$-after RFA have been used with Sonazoid CEUS to evaluate the post-RFA response.

\section{Follow-up after tumor ablation}

CEUS is very useful to identify LTP after tumor ablation [5]. A suspicious area within the ablation zone can be confirmed to have viable tumor using defect reperfusion imaging. However, CEUS cannot replace CT or MRI for routine follow-up after tumor ablation as it does not allow assessment of both intra- and extra-hepatic tumor recurrence [10].

\section{Combined fusion imaging and contrast-enhanced ultrasound}

High-end US machines from many manufacturers provide fusion imaging technology, and most of these are based on electromagnetic tracking systems, which consist of a magnetic field generator, a position sensor, and a position sensor unit [158]. If a US machine capable of fusion imaging is available, it can be utilized for localizing small inconspicuous tumors by using a reference dataset such as preacquired CT/MR images [158]. Many studies have reported that fusion imaging can improve tumor visibility and technical feasibility of RFA for small HCCs [159-163].

CEUS can be combined with fusion imaging for localizing small HCCs with poor lesion conspicuity. With fusion imaging, most small tumors can be localized [164]. Even though the tumor is not identified on fusion imaging, the operator can estimate the location of the target lesion based on fused CT/MR images before injecting contrast agent. Therefore, tumoral enhancement in the arterial phase, which is characterized by a short temporal window, may be observed in many cases. If arterial enhancement is found with the initial injection of Sonazoid and the lesion is seen as a defect in the 
postvascular phase, it is not necessary to reinject Sonazoid for defect reperfusion imaging. Therefore, the overall procedure time of CEUSguided interventional procedures can be reduced $[158,165]$.

Min et al. [165] reported that $83.3 \%$ (25/30) of small HCCs (mean, $1.2 \mathrm{~cm}$; range, 0.6 to $1.7 \mathrm{~cm}$ ) inconspicuous on fusion imaging became conspicuous after adding Sonazoid CEUS to fusion imaging. Among the tumors $(n=23)$ in which RFA was considered feasible, the rates of technical success and major complication were $91.3 \%$ (21/23) and 4.3\% (1/23), respectively [165].

Recurrent subcentimeter HCCs can also benefit from percutaneous fusion imaging-guided RFA, with the use of CEUS as a problemsolving tool. For recurrent subcentimeter HCCs, Song et al. [166] reported that the feasibility rate of percutaneous RFA at planning US was $65.7 \%(138 / 210)$. It is noteworthy that the rate of both technical success and technique efficacy was $98.4 \%(123 / 125)$ in that study. The cumulative LTP rate at 3 years was $7.4 \%$, and the major complication rate was $2.5 \%$ (3/119) [166]. Therefore, local ablation therapy under fusion imaging and/or CEUS guidance seems to be promising as it is effective and safe for the management of small HCCs [166-168].

However, not all small tumors can be localized by fusion imaging and/or CEUS as the detectability at US is intrinsically limited by the sonographic window and the location and size of the target lesion. Given that MR guidance has several advantages, such as the near real-time guiding capability of MR fluoroscopic imaging, higher sensitivity in detecting small hepatic lesions, ability to monitor tissue temperature during ablation, free selection of imaging planes, and absence of ionizing radiation hazards $[169,170]$, MRI may be used as an alternative guiding modality for small HCCs inconspicuous on fusion imaging and/or CEUS [167]. However, MR-compatible devices are required for MR-guided ablation procedures.

\section{CQ13: Is Sonazoid CEUS helpful to perform local ablation therapy for liver tumors?}

Consensus Statement \#13

Sonazoid CEUS improves tumor visibility and technical feasibility of local ablation therapy and thereby leads to favorable local tumor control for small HCCs inconspicuous in B-mode US. (A-1)

(Agreement level 9.5, evidence level 9.5, recommendation level 9.3)

\section{CQ14: Is Sonazoid CEUS useful to assess treatment response and complications at the end of local ablation therapy?}

Consensus Statement \#14

Early treatment response and presence of postablation complications can be assessed with CEUS at the end of local ablation therapy. (B-2) (Agreement level 8.4, evidence level 7.2, recommendation level 7.7)

\section{CQ15: Is combined fusion imaging and CEUS-guided local} ablation effective for small hepatic tumors?

Consensus Statement \#15

Combined fusion imaging and CEUS-guided local ablation is effective for the management of hepatic tumors inconspicuous on B-mode US. (B-1)

(Agreement level 8.5, evidence level 8.2, recommendation level 8.7)

\section{Chapter 5: Quantitative Contrast-Enhanced Ultrasound}

\section{Monitoring Treatment Response \\ Background}

Locoregional therapies, which conventionally include ablation, whatever the modality used, and transarterial chemoembolization (TACE), play a key role in the management of patients with HCC $[75,171,172]$. For systemic therapy, CEUS may enable early prediction and monitoring of treatment response [173].

\section{Monitoring treatment response for locoregional treatments such as radiofrequency ablation or transarterial chemoembolization}

Unenhanced US is commonly used to guide ablation. It is easy to use and widely available; however, even when combined with Doppler, it does not provide useful information on the extent of the ablation. Assessment of the tissue perfusion is crucial to differentiate necrotic from viable residual tumor.

The addition of Sonazoid in the arterial phase and/or defect reperfusion imaging can provide important information on the residual viable tumor $[5,147,151,156,174]$. In particular, defect reperfusion imaging is very useful for detecting a residual tumor or local recurrence after locoregional treatment, because Sonazoid CEUS is less affected by the observer's experience and is more accurate in the diagnosis of local recurrence after treatment for HCC than dynamic CT $[171,172,175]$.

\section{Monitoring treatment response for systemic therapy}

The Response Evaluation Criteria in Solid Tumors (RECIST) guidelines for the assessment of tumor response are no longer considered adequate for locoregional treatment because of the poor relationship between necrosis and tumor size [176]. Accordingly, the RECIST criteria have been amended, at least for HCC [177].

Since antiangiogenic treatment frequently induces necrosis without causing tumor shrinkage, functional imaging techniques are particularly suitable for early assessment of response, a task for which the RECIST criteria are unsatisfactory.

Studies of various types of tumors treated with antiangiogenic 
therapies have confirmed that dynamic CEUS (other than Sonazoid) may enable early prediction of response to treatment [20]. However, the benefits of Sonazoid CEUS were recently reported, including its usefulness for evaluation and early detection of major adverse events [173]. It has been reported that analysis of the TIC and arrival time parametric imaging were useful for evaluating early response to sorafenib for advanced HCC $[173,178-182]$. Although Sonazoid enables the evaluation of vascularity during systemic therapy that cannot be detected by CECT or contrast-enhanced MRI, Sonazoid may only be useful to evaluate treatment response if the number of HCCs is less than two or three.

\section{CQ16: Is Sonazoid CEUS useful to evaluate the monitoring treatment response after locoregional therapies (RFA, TACE, etc.)?}

Consensus Statement \#16

Sonazoid CEUS is useful to provide important information in monitoring after locoregional therapies (RFA, TACE, etc.). (B-1)

(Agreement level 8.5, evidence level 8.6, recommendation level 8.6)

\section{CQ17: Is Sonazoid CEUS useful in monitoring treatment response after systemic therapy?}

Consensus Statement \#17

Sonazoid CEUS may be useful in monitoring treatment response after systemic therapy for a limited number of lesions. (B-2)

(Agreement level 8.1, evidence level 7.0, recommendation level 7.5)

\section{Others (Fibrosis, etc.) \\ Background}

Liver function is regulated by four factors: uptake, metabolism, secretion by the liver cells, and sinusoidal blood flow [183]. On the other hand, blood flow within the liver parenchyma is strongly affected by histological changes such as liver fibrosis, edema, and inflammation [33]. If the disease has progressed to liver cirrhosis, blood flow into the sinusoids from the portal vein is reduced and compensated for by arterial blood (arterialization) [184]. Therefore, analysis of hemodynamics within the liver parenchyma may become a relevant method for assessing liver histology and function.

\section{Arrival time parametric imaging}

The color of each pixel within an arrival time parametric image is encoded as red or yellow according to its arrival time: if earlier than 5 seconds, red; and if later than 5 seconds, yellow. Several studies suggested that arrival time parametric images can depict the histological evolution, especially the grade of liver fibrosis, seen in different chronic liver diseases $[108,185,186]$.

\section{Perfusion parametric imaging}

The pixel color coding in a perfusion parametric image is determined with respect to portal vein arrival time: pixels with an arrival time earlier than portal arrival time are coded as red, and if later than portal arrival time, as blue. When the liver parenchyma becomes reddominant, this indicates the arterialization of blood flow within the liver parenchyma. Marked arterialization is generally only observed in patients with liver cirrhosis [185].

\section{FLASH imaging}

FLASH imaging is performed to burst microbubbles accumulated in the liver parenchyma by high-power pulses in the postvascular phase at 10 minutes after Sonazoid administration. FLASH images are used to measure the distance between the liver surface and collapsed microbubbles. It has been suggested that FLASH images obtained in the postvascular phase may enable comprehensive and noninvasive assessment of pathologic changes related to liver disease progression; such assessments may include not only staging of liver fibrosis but also estimation of Kupffer cell function in patients with chronic liver disease [187].

\section{Chapter 6: Other Organs}

\section{Contrast-Enhanced Ultrasound for Pancreatobiliary Disease}

A few retrospective studies have reported the use of Sonazoid CEUS in diagnosis of pancreatobiliary disease. Three-dimensional contrastenhanced patterns are useful for the differential diagnosis of solid pancreatic lesions, with a reported diagnostic accuracy of $90.5 \%$ [188]. For the gallbladder, Sonazoid CEUS showed $94 \%$ sensitivity, $89 \%$ specificity, and $92 \%$ accuracy in the differential diagnosis of malignant and benign polypoid lesions [189], and $85.2 \%$ accuracy in the diagnosis of gangrenous cholecystitis [190].

\section{Contrast-Enhanced Endoscopic Ultrasound for Pancreas Background}

Most pancreatic lesions, including carcinomas, neuroendocrine neoplasms, and inflammatory tumors, exhibit low-echoic solid masses on conventional endoscopic ultrasound (EUS). It is sometimes difficult to differentiate carcinomas from other tumors among the low echoic solid masses depicted on the conventional EUS. Contrast harmonic imaging can detect signals from microbubbles in the vessels with very slow flow without Doppler-related artifacts. With first-generation UCAs such as Levovist (Schering AG, Berlin, Germany), a contrast harmonic imaging technique was not available for EUS because the transducer of the echoendoscope was too small to produce enough acoustic power for contrast harmonic imaging. Second-generation UCAs such as SonoVue (Bracco Imaging, Milan, 
Italy) and Sonazoid produce harmonic signals at lower acoustic power and are therefore suitable for EUS imaging at low acoustic power $[191,192]$. Several studies have evaluated the utility of contrast-enhanced EUS (CE-EUS) for diagnosing solid lesions in the pancreas.

\section{Strength of evidence}

Basic B-mode EUS is limited in its ability to characterize pancreatic solid lesions because it depicts various kinds of tumors as low echoic masses. Contrast enhancement improves the ability to discriminate ductal carcinomas from other tumors [193]. The characterization of pancreatic solid masses is generally based on a qualitative assessment of the degree of enhancement, compared with the surrounding tissue. The typical pattern of ductal carcinomas on CE-EUS is hypoenhancement (lower echo intensity of the tumor than the surrounding tissue), while the typical pattern of inflammatory mass and neuroendocrine tumors is isoenhancement and hyperenhancement, respectively [194].

There are two published meta-analyses on the role of contrast-enhanced EUS for the diagnosis of solid lesions in the pancreas. Gong et al. [195] analyzed CE-EUS for the diagnosis of adenocarcinoma and the pooled sensitivity and specificity were $94 \%(91 \%-95 \%)$ and specificity $89 \%(85 \%-92 \%)$ respectively. However, this study included not only CE-EUS but also contrastenhanced Doppler EUS.

Yamashita et al. [196] focused on CE-EUS and reported that its sensitivity and specificity for differentiating ductal carcinomas from the other tumors with CE-EUS were $93 \%$ and $80 \%$, respectively. Regarding CE-EUS with Sonazoid, Kitano et al. [197] reported that CE-EUS-depicted hypoenhancement diagnoses ductal carcinomas with a sensitivity and specificity of $95.1 \%(92.7 \%-96.7 \%)$ and $89.0 \%(83.0 \%-93.1 \%)$, respectively [197]. For diagnosing small $(\leq 2 \mathrm{~cm})$ carcinomas by CE-EUS, the sensitivity and specificity were $91.2 \%(82.5 \%-95.1 \%)$ and $94.4 \%(86.2 \%-98.1 \%)$, respectively. CE-EUS is superior to multidetector CT (MDCT) in diagnosing small $(\leq 2 \mathrm{~cm})$ carcinomas $(P<0.05)$. CE-EUS-depicted hypervascular enhancement diagnoses neuroendocrine tumors with a sensitivity and specificity of $78.9 \%(61.4 \%-89.7 \%)$ and $98.7 \%(96.7 \%-$ $98.8 \%$ ), respectively. The recent development of software for TIC analysis enables some degree of quantitative assessment [198-201].

CQ18: Is CE-EUS with Sonazoid useful for characterization of solid lesions in the pancreas?

Consensus Statement \#18

CE-EUS with Sonazoid is useful for the characterization of solid lesions in the pancreas. (A-1)

(Agreement level 9.1, evidence level 8.5, recommendation level 8.8)

\section{CQ19: Is CE-EUS useful for differentiation of malignant cysts from benign cysts in the pancreas?}

Consensus Statement \#19

CE-EUS can be recommended to differentiate malignant from benign cysts in the pancreas. (B-2)

(Agreement level 8.3, evidence level 6.9, recommendation level 7.8)

\section{Contrast-enhanced endoscopic ultrasound for gallbladder Background}

Differential diagnosis of gallbladder lesions is challenging. Gallbladder lesions include sludge, cholesterol polyps, adenomyomatosis, and cancer. These lesions sometimes appear similar on ultrasonographic images. To resolve this, several studies have evaluated the utility of CE-EUS for diagnosing gallbladder lesions [202-206].

\section{Study procedure}

For evaluation of gallbladder lesions, Sonovue or Sonazoid is used. After contrast agent infusion, vascular and enhancement patterns are assessed by the examination of continuous $0-15$ seconds (vascular images) and 40-60 seconds (perfusion images) images, respectively [202].

\section{Contrast-enhanced endoscopic ultrasound for gallbladder lesions}

Gallbladder sludge and gallstones show the absence of enhancement on CE-EUS, whereas other gallbladder lesions appear enhanced in almost all cases $[202,203]$. Kamata et al. [202] evaluated 125 patients with localized gallbladder lesions using CEEUS. In this study, the positive predictive value (PPV) of cancer with spotty vessels, no vessels, and irregular vessels in the vascular phase images of CE-EUS was $15 \%, 0 \%$, and $95 \%$, respectively. In contrast, the PPV of cancer with homogeneous, absent, homogeneous with clear perfusion defects, and heterogeneous enhancement in the CEEUS perfusion images was $5.9 \%, 3.3 \%, 0 \%$, and $97 \%$, respectively. Irregular vessel patterns in the vascular images and heterogeneous enhancement in the perfusion images are the significant predictive factors for gallbladder cancer. In one study, irregular vessels and/ or heterogeneous patterns on the CE-EUS predicted malignancy with sensitivity, specificity, and accuracy of $90 \%, 98 \%$, and $96 \%$, respectively [202]. Choi et al. [203] assessed 93 patients with gallbladder polyps larger than $10 \mathrm{~mm}$ using CE-EUS. They also found that irregular vessels and perfusion defects (equivalent to heterogeneous enhancement) were the signs of malignancy. The sensitivity, specificity, PPV, and negative predictive value of the CE-EUS for diagnosing malignant gallbladder polyps were $93.5 \%, 93.2 \%, 87.8 \%$, and $96.5 \%$, respectively. Moreover, the 
management plan was changed after CE-EUS in 8 out of 93 (8.6\%) patients. Imazu et al. [204] evaluated the utility of CE-EUS for the differential diagnosis of gallbladder wall thickening in 36 patients. In this study, the overall sensitivity, specificity, and accuracy for diagnosing malignant gallbladder wall thickening of conventional EUS versus CE-EUS were $83.3 \%$ versus $89.6 \%, 65 \%$ versus $98 \%$, and $73.1 \%$ versus $94.4 \%$, respectively. The authors reported that heterogeneous distribution pattern of the contrast agent on CEEUS was significantly linked to the wall thickening of gallbladder carcinoma with 0.94 of the AUC. Sugimoto et al. [205] evaluated 35 patients who underwent CE-EUS for gallbladder lesions 10 $\mathrm{mm}$ or more in diameter. In this study, a brindled enhancement pattern (equivalent to heterogeneous enhancement) was considered a sign of malignancy. As evaluated by three inexperienced endosonographers, the overall sensitivity, specificity, and accuracy of CE-EUS for diagnosing gallbladder cancer were $76.1 \%, 66.7 \%$, and $69.4 \%$, respectively, which were lower than reported in other studies. This result indicates that an experienced endosonographer is needed to evaluate the CE-EUS findings. Leem et al. [206] evaluated 35 cases of gallbladder masses and reported that malignant masses tended to show heterogeneous patterns both on conventional EUS and CE-EUS. When CE-EUS was used in addition to conventional EUS for diagnosing malignant masses, the sensitivity increased to $97.1 \%$ from $77.1 \%$, but the specificity decreased to $55.5 \%$ from $82.7 \%$. They also performed a univariate analysis of predictive variables for gallbladder malignant masses and found that the odds ratio for malignancy of heterogeneous patterns on conventional EUS was 16.1 while that on CE-EUS was 42.3 .

Taken together, these studies strongly suggest that irregular vessels and heterogeneous patterns on the CE-EUS are the significant predictive factors for gallbladder cancer. Based upon these findings, CE-EUS may improve diagnostic accuracy for malignant gallbladder lesions. However, CE-EUS is not necessary in the majority of cases and has not been widely used. Moreover, further prospective studies involving larger numbers of cases are warranted.

CQ20: Is CE-EUS useful for differential diagnosis of biliary sludge from polypoid gallbladder lesions?

Consensus Statement \#20

CE-EUS can be used for differential diagnosis of biliary sludge from polypoid gallbladder lesions. (B-2)

(Agreement level 8.0, evidence level 7.1, recommendation level 7.9)

CQ21: Is CE-EUS useful for differential diagnosis of benign and malignant gallbladder lesions?

Consensus Statement \#21
CE-EUS can be used for differentiating between benign and malignant gallbladder lesions. (B-2)

(Agreement level 7.6, evidence level 7.0, recommendation level 7.5)

\section{Chapter 7: Others}

\section{Limitations}

Similar to other US imaging techniques, Sonazoid-enhanced CEUS shows limitations under the following conditions: poor acoustic window caused by the presence of bowel gas or abundant fatty tissue in the US path; insufficient US transmission due to deepseated, narrow intercostal space, or obese body habitus; and movement during scanning $[43,207]$. In addition, it is also limited within blind spots such as the liver dome or lateral part of the liver, similar to other US imaging techniques, even though this problem can be reduced by postural change [45]. Other limitations are the technique and experience of a CEUS operator, which influences diagnosis accuracy. For this reason, CEUS training and standardization of CEUS examination are important.

\section{Artifacts}

\section{Pseudoenhancement artifact}

In some cases involving strongly echogenic lesions, the echogenic interface is not completely canceled in nonlinear imaging so that it is seen on contrast agent imaging and is difficult to separate from microbubble signals. Examples of strongly echogenic lesions include calcifications, AMLs and hemangiomas, and air bubbles produced during thermal ablation. These are attributed to the ineffective subtraction of nonlinear harmonic signals of these echogenic lesions and the echogenic interface [208]. This artifact can be suppressed by adjusting power and gain settings to the proper level. When there is a need to differentiate this artifact from true contrast signals, a high MI flash imaging can be helpful. After the flash, true enhancement will be lost and reperfusion will occur, whereas a pseudoenhancement artifact will persist regardless of the flash.

\section{Pseudowash-out artifact}

This artifact is related to the accelerated bubble destruction when the transducer is held fixed in the same location. A slowly enhancing lesion can show this phenomenon due to unintended bubble destruction when the transducer remains in a stationary position for a long time and may lead to misdiagnosis. Lowering the frame rate, intermittent imaging, placing the focal zone beyond the area of interest, and sweeping through a lesion may help avoid this artifact [208]. 


\section{Veiling artifacts (or veiling effect)}

Sonazoid has a unique artifact known as a "veiling artifact" [209]. The veiling artifact appears on B-mode images in the postvascular phase (Kupffer phase). This artifact is seen as an echogenic wavy line that moves inferiorly over a sequence of images. It may be because Sonazoid microbubbles with shell stiffer than other UCAs are densely taken up by Kupffer cells during the postvascular phase (Kupffer phase).

\section{Safety Considerations}

\section{Basic safety considerations: cavitation and its biological effects}

The effects of US irradiation on the biological tissues are categorized into thermal effects and mechanical (or nonthermal) effects. The use of UCAs may induce additional effects caused by both thermal and mechanical mechanisms, although the mechanical effect is dominant.

The phenomenon of bubbles undergoing volumetric oscillation under the condition of pressure fluctuation is known as cavitation. This is an important phenomenon that brings about a wide range of biological effects by various mechanisms. Stable oscillation of the bubbles under conditions of relatively low pressure is called noninertial (stable) cavitation, while violent oscillation that causes collapse and fragmentation of bubbles is called inertial (transient) cavitation.

By nature, there are no bubbles in the human tissues. Highintensity long-duration US is required to induce inertial cavitation for therapeutic applications. Short-duration diagnostic US pulses normally do not induce cavitation; however, it is known that irradiation of pulmonary alveoli or gas bubbles in the intestine with such pulses can induce the mechanical effects of cavitation, and basic studies indicate the possibility of UCA microbubbles showing similar behavior on exposure to diagnostic US $[210,211]$. Clinical practitioners should be aware that the use of UCAs in CEUS distributes microbubbles inside the body, thus increasing the possibility of inertial cavitation, and therefore increases the likelihood of bioeffects.

\section{Reduction of cavitation effects}

The WFUMB Safety Committee describes a number of strategies to reduce the likelihood of bioeffects [212].

- Reduction of UCA dose

- Imaging with lower MI

- Imaging with higher US frequency

- Reduction of the duration of examination

- Adjusting the timing of cardiac triggering in cardiac application The WFUMB Safety Committee recommends that the use of a
UCA should be avoided 24 hours before extracorporeal shock wave therapy $[213,214]$. Additional care should be taken when longer duration pulses for acoustic radiation force impulse (ARFI) imaging are used.

\section{Clinical safety consideration: adverse events}

Of the 397 subjects evaluated in the preapproval Sonazoid studies in Korea, there were $25(6.3 \%)$ reported adverse events. The most commonly noted adverse events were diarrhea $(1.0 \%, n=4)$, headache $(1.0 \%, n=4)$, proteinuria $(0.8 \%, n=3)$, neutropenia $(0.5 \%, n=2)$, exanthema $(0.5 \%, n=2)$, dry mouth $(0.5 \%, n=2)$, and injection site discomfort $(0.5 \%, n=2)$ at the time of Sonazoid approval [215].

According to the postmarketing studies involving 3,066 patients in Korea, the adverse event rate was 3.2\% (99/3,066 patients, 138 events), regardless of relevancy [215]. The most commonly noted adverse events were the elevation of aspartate aminotransferase (more than 1\%, less than 10\%). Elevation of alanine aminotransferase, bilirubin, and C-reactive protein, abdominal pain, nausea, and urticaria was reported to be more than $0.1 \%$ and less than $1 \%$. The rare adverse events $(<0.1 \%)$ were chill, general weakness, back pain, fatigue, indigestion, dizziness, sensory abnormality, pneumonia, and falling down. Among them, chill, indigestion, abdominal pain, nausea, and urticaria were deemed to be at least possibly related to Sonazoid.

A recent diagnostic trial in Korea evaluated adverse events in 524 patients [78]. The adverse event rate was 5.7\% (30/524; $95 \% \mathrm{Cl}, 3.9 \%$ to $8.1 \%$ ). There were 39 adverse events (Common Terminology Criteria for Adverse Events [CTCAE] grade 1, 32 events; grade 2, 7 events), which were judged to be possibly $(n=15)$, probably $(n=16)$, or definitely $(n=8)$ associated with the administration of perfluorobutane. All participants recovered without specific treatment. None of the participants suffered longterm or serious (CTCAE grade 3 or more) adverse events. The most commonly noted adverse events were diarrhea $(2.1 \%, n=11)$, headache $(1.9 \%, n=10)$, dyspepsia $(0.8 \%, n=4)$, abdominal pain $(0.6 \%, n=3)$, chills $(0.4 \%, n=2)$, hot flush $(0.4 \%, n=2)$, and nausea $(0.4 \%, n=2)$ at the time of approval.

According to postmarketing studies involving 3,422 patients in Japan, there were $0.5 \%$ (17 cases among 3,422 patients) adverse events, regardless of relevancy [216]. A Japanese multicenter trial reported an adverse drug reaction rate of $10.4 \%(95 \% \mathrm{Cl}$, $6.1 \%$ to $14.7 \%$ ) (20 cases among 193 patients) [217]. The most common adverse events were albuminuria 1.6\% (3 cases among 193 patients) and diarrhea 1.6\% (3 cases among 193 patients). All adverse drug reactions were mild. No deaths and serious or severe adverse events were reported in this study. 
Shock and anaphylactoid reactions may occur even though there have been no reports of these to date. Therefore, patients should be carefully monitored, and appropriate measures should be taken if any abnormal events such as dyspnea, hypotension, or rash are noticed.

\section{Recommendation statements}

1. Basic studies indicate that it is possible for diagnostic US exposure in the presence of microbubbles to cause adverse or therapeutic effects to biological tissues

2. To reduce adverse effects, CEUS examinations should be carried out using a lower dose of the agent, lower MI with higher frequency, and examination times that are As Short As Reasonably Achievable for obtaining essential diagnostic information, according to the As Low As Reasonably Achievable principle

3. Use of a UCA should be avoided 24 hours before extracorporeal shock wave therapy, and additional care should be taken when longer duration pulses for ARFI imaging are used

\section{Contraindications}

Sonazoid is contraindicated in patients with a history of hypersensitivity to the active substance or to any of the excipients (sucrose, perfluorobutane, and H-EPSNa) [218]. The possibility of hypersensitivity, including serious, life-threatening anaphylactoid reaction or anaphylactoid shock should always be considered [218]. Advanced life support facilities should be readily available.

For the following cases, special warnings and precautions for use are required [218]. Sonazoid contains a chicken egg-derived surfactant (H-EPSNa). In patients with a history of allergy to eggs or egg products, Sonazoid should be used only if the benefit clearly outweighs the potential risk. Care should be taken in patients with right-to-left arteriovenous cardiac or pulmonary shunt, as Sonazoid enters the circulation directly without passing through the lungs.

\begin{tabular}{|c|c|}
\hline AFSUMB & $\begin{array}{l}\text { Asian Federation of Societies for Ultrasound in Medicine } \\
\text { and Biology }\end{array}$ \\
\hline ACUCl & Asian congress of ultrasound contrast imaging \\
\hline ALT & Alanine aminotransferase \\
\hline AML & Angiomyolipoma \\
\hline ARFI & Acoustic radiation force impulse \\
\hline AST & Aspartate aminotransferase \\
\hline AUC & Area under the curve \\
\hline CE-EUS & Contrast-enhanced EUS \\
\hline CEUS & Contrast-enhanced ultrasound \\
\hline CM & Confluent multinodular type \\
\hline
\end{tabular}

\begin{tabular}{|c|c|}
\hline $\mathrm{CT}$ & Computed tomography \\
\hline CTCAE & Common terminology criteria for adverse events \\
\hline CTSUM & Chinese Taipei Society of Ultrasound in Medicine \\
\hline DN & Dysplastic nodule \\
\hline DWI & Diffusion-weighted MR image \\
\hline EFSUMB & $\begin{array}{l}\text { European Federation of Societies for Ultrasound in } \\
\text { Medicine and Biology }\end{array}$ \\
\hline EUS & Endoscopic ultrasound \\
\hline FLL & Focal liver lesion \\
\hline $\mathrm{FNH}$ & Focal nodular hyperplasia \\
\hline FWHM & Full width of half the maximum \\
\hline Gd-EOB-DTPA & $\begin{array}{l}\text { Gadolinium ethoxybenzyl diethylenetriamine pentaacetic } \\
\text { acid (=gadoxetic acid) }\end{array}$ \\
\hline $\mathrm{HCA}$ & Hepatocellular adenoma \\
\hline $\mathrm{HCC}$ & Hepatocellular carcinoma \\
\hline H-EPSNa & Hydrogenated egg phosphatidylserine sodium \\
\hline HNF & Hepatocyte nuclear factor \\
\hline ICC & Intrahepatic cholangiocarcinoma \\
\hline IOUS & Intraoperative ultrasound \\
\hline ISUCI & $\begin{array}{l}\text { International Symposium on Ultrasound Contrast } \\
\text { Imaging }\end{array}$ \\
\hline JSUM & Japanese Society of Ultrasound in Medicine \\
\hline KSUM & Korean Society of Ultrasound in Medicine \\
\hline $\mathrm{Ml}$ & Mechanical index \\
\hline MRI & Magnetic resonance imaging \\
\hline $\mathrm{Pl}$ & Peak intensity \\
\hline PVT & Portal vein thrombosis \\
\hline RFA & Radiofrequency ablation \\
\hline RN & Regenerating nodule \\
\hline RT & Rise time \\
\hline SN & Single nodular type \\
\hline SNEG & Single nodular type with extranodular growth \\
\hline SPIO & Superparamagnetic iron oxide \\
\hline TIC & Time intensity curve \\
\hline TTP & Time to peak \\
\hline UCA & Ultrasound contrast agent \\
\hline US & Ultrasound \\
\hline WFUMB & World Federation for Ultrasound in Medicine and Biology \\
\hline WiAUC & AUC during wash-in \\
\hline WiWoAUC & WiAUC plus WoAUC \\
\hline WoAUC & AUC during washout \\
\hline
\end{tabular}

ORCID: Jae Young Lee: https://orcid.org/0000-0001-6946-6042; Yasunori Minami: https://orcid.org/0000-0001-7146-5954; Byung Ihn Choi: https://orcid.org/00000002-5613-1881; Won Jae Lee: https://orcid.org/0000-0001-5833-989X; Yi-Hong Chou: https://orcid.org/0000-0002-7418-4514; Woo Kyoung Jeong: https://orcid.org/00000002-0676-2116; Mi-Suk Park: https://orcid.org/0000-0001-5817-2444; Nobuki Kudo: 
https://orcid.org/0000-0003-3463-3714; Min Woo Lee: https://orcid.org/0000-00019048-9011; Ken Kamata: https://orcid.org/0000-0003-1568-0769; Hiroko lijima: https://orcid.org/0000-0002-5841-8827; So Yeon Kim: https://orcid.org/0000-00016853-8577; Kazushi Numata: https://orcid.org/0000-0003-4671-4431; Katsutoshi Sugimoto: https://orcid.org/0000-0001-6271-7806; Hitoshi Maruyama: https://orcid.org/00000003-3371-3157; Yasukiyo Sumino: https://orcid.org/0000-0002-1044-8808; Chikara Ogawa: https://orcid.org/0000-0002-4534-6692; Masayuki Kitano: https://orcid.org/00000001-6885-9223; ljin Joo: https://orcid.org/0000-0002-1341-4072; Junichi Arita: https://orcid.org/0000-0003-1929-8479; Ja-Der Liang: https://orcid.org/0000-00024532-0118; Hsi-Ming Lin: https://orcid.org/0000-0001-7527-6181; Christian Nolsoe: https://orcid.org/0000-0003-0168-6131; Odd Helge Gilja: https://orcid.org/00000002-0436-0383; Masatoshi Kudo: https://orcid.org/0000-0002-4102-3474

\section{※ Author affiliations}

'Department of Radiology, Seoul National University Hospital, Seoul, Korea; ${ }^{2}$ Department of Gastroenterology and Hepatology, Faculty of Medicine, Kindai University, Higashi-Osaka, Japan; ${ }^{3}$ Department of Radiology, Chung Ang University Hospital, Seoul; ${ }^{4}$ Department of Radiology and Center for Imaging Science, Samsung Medical Center, Sungkyunkwan University School of Medicine, Seoul, Korea; ${ }^{5}$ Department of Medical Imaging and Radiological Technology, Yuanpei University of Medical Technology, Hsinchu; ${ }^{6}$ Department of Radiology, National Yang Ming University, Taipei, Taiwan; 'Department of Radiology, Severance Hospital, Yonsei University College of Medicine, Seoul, Korea; ${ }^{8}$ Laboratory of Biomedical Engineering, Graduate School of Information Science and Technology, Hokkaido University, Sapporo; ${ }^{9}$ Department of Ultrasound, Hepatobiliary and Pancreatic Disease, Hyogo College of Medicine, Nishinomiya, Japan; ${ }^{10}$ Department of Radiology, University of Ulsan College of Medicine, Asan Medical Center, Seoul, Korea; ${ }^{11}$ Gastroenterological Center, Yokohama City University Medical Center, Yokohama; ${ }^{12}$ Department of Gastroenterology and Hepatology, Tokyo Medical University, Tokyo; ${ }^{13}$ Department of Gastroenterology, Juntendo University, Tokyo; ${ }^{14}$ Department of Gastroenterology and Hepatology, Toho University Medical Center, Tokyo; ${ }^{15}$ Department of Gastroenterology and Hepatology, Takamatsu Red Cross Hospital, Takamatsu; ${ }^{16}$ Department of Gastroenterology and Hepatology, Wakayama Medical University Hospital, Wakayama; ${ }^{17}$ Hepato-Biliary-Pancreatic Surgery Division and Artificial Organ and Transplantation Division, Department of Surgery, Graduate School of Medicine, The University of Tokyo, Tokyo, Japan; ${ }^{18}$ Department of Gastroenterology and Hepatology, National Taiwan University, Taipei; ${ }^{19}$ Department of Gastroenterology and Hepatology, Chang Gung University, Taipei, Taiwan; ${ }^{20}$ Ultrasound Section, Division of Surgery, Department of Gastroenterology, Herlev Hospital, Copenhagen Academy for Medical Education and Simulation, University of Copenhagen, Copenhagen, Denmark; ${ }^{21}$ National Centre for Ultrasound in Gastroenterology, Haukeland
University Hospital, Bergen; ${ }^{22}$ Department of Clinical Medicine, University of Bergen, Bergen, Norway

\section{Author Contributions}

Conceptualization: Lee JY, Minami Y, Choi BI, Lee WJ, Kudo M. Data acquisition: Lee JY, Minami Y. Data analysis or interpretation: all authors. Drafting of the manuscript: Lee JY, Minami Y, Choi BI, Kudo M. Critical revision of the manuscript: Lee JY, Minami Y, Choi BI, Lee WJ, Jeong WK, Park MS, Kudo N, Lee MW, Joo I, Kudo M. Approval of the final version of the manuscript: all authors.

\section{Conflict of Interest}

No potential conflict of interest relevant to this article was reported.

\section{Acknowledgments}

Financially supported by AFSUMB.

\section{References}

1. Guyatt $G H$, Oxman AD, Kunz R, Falck-Ytter $Y$, Vist GE, Liberati $A$, et al. Going from evidence to recommendations. BMJ 2008;336:10491051.

2. Yanagisawa K, Moriyasu F, Miyahara T, Yuki M, lijima H. Phagocytosis of ultrasound contrast agent microbubbles by Kupffer cells. Ultrasound Med Biol 2007;33:318-325.

3. Shunichi S, Hiroko I, Fuminori M, Waki H. Definition of contrast enhancement phases of the liver using a perfluoro-based microbubble agent, perflubutane microbubbles. Ultrasound Med Biol 2009;35:1819-1827.

4. Watanabe R, Matsumura M, Munemasa T, Fujimaki M, Suematsu $M$. Mechanism of hepatic parenchyma-specific contrast of microbubble-based contrast agent for ultrasonography: microscopic studies in rat liver. Invest Radiol 2007:42:643-651.

5. Kudo M, Hatanaka K, Maekawa K. Newly developed novel ultrasound technique, defect reperfusion ultrasound imaging, using Sonazoid in the management of hepatocellular carcinoma. Oncology 2010;78 Suppl 1:40-45.

6. Kudo M, Hatanaka K, Kumada T, Toyoda H, Tada T. Double-contrast ultrasound: a novel surveillance tool for hepatocellular carcinoma. Am J Gastroenterol 2011;106:368-370.

7. Albrecht T, Blomley M, Bolondi L, Claudon M, Correas JM, Cosgrove $D$, et al. Guidelines for the use of contrast agents in ultrasound. January 2004. Ultraschall Med 2004;25:249-256.

8. Claudon M, Cosgrove D, Albrecht T, Bolondi L, Bosio M, Calliada F, et al. Guidelines and good clinical practice recommendations for contrast enhanced ultrasound (CEUS) - update 2008. Ultraschall Med 2008;29:28-44.

9. Claudon M, Dietrich CF, Choi BI, Cosgrove DO, Kudo M, Nolsoe CP, 
et al. Guidelines and good clinical practice recommendations for contrast enhanced ultrasound (CEUS) in the liver: update 2012: a WFUMB-EFSUMB initiative in cooperation with representatives of AFSUMB, AIUM, ASUM, FLAUS and ICUS. Ultraschall Med 2013;34:11-29.

10. Claudon M, Dietrich CF, Choi BI, Cosgrove DO, Kudo M, Nolsoe CP, et al. Guidelines and good clinical practice recommendations for Contrast Enhanced Ultrasound (CEUS) in the liver: update 2012: a WFUMB-EFSUMB initiative in cooperation with representatives of AFSUMB, AIUM, ASUM, FLAUS and ICUS. Ultrasound Med Biol 2013;39:187-210.

11. Nolsoe $C P$, Lorentzen T. International guidelines for contrastenhanced ultrasonography: ultrasound imaging in the new millennium. Ultrasonography 2016;35:89-103.

12. PubMed [Internet]. Bethesda, MD: National Center for Biotechnology Information, 2019 [cited 2019 May 7]. Available from: http://www.ncbi.nlm.nih.gov/pubmed/.

13. Abbott JG. Rationale and derivation of MI and TI: a review. Ultrasound Med Biol 1999;25:431-441.

14. Duck FA. Nonlinear acoustics in diagnostic ultrasound. Ultrasound Med Biol 2002;28:1-18.

15. de Jong N, Frinking PJ, Bouakaz A, Ten Cate FJ. Detection procedures of ultrasound contrast agents. Ultrasonics 2000;38:8792.

16. Quaia E. Microbubble ultrasound contrast agents: an update. Eur Radiol 2007;17:1995-2008.

17. Simpson DH, Chin CT, Burns PN. Pulse inversion Doppler: a new method for detecting nonlinear echoes from microbubble contrast agents. IEEE Trans Ultrason Ferroelectr Freq Control 1999;46:372382.

18. Greis C. Technical aspects of contrast-enhanced ultrasound (CEUS) examinations: tips and tricks. Clin Hemorheol Microcirc 2014;58:89-95.

19. Tranquart F, Mercier L, Frinking P, Gaud E, Arditi M. Perfusion quantification in contrast-enhanced ultrasound (CEUS): ready for research projects and routine clinical use. Ultraschall Med 2012;33 Suppl 1:S31-S38.

20. Dietrich CF, Averkiou MA, Correas JM, Lassau N, Leen E, Piscaglia F. An EFSUMB introduction into dynamic contrast-enhanced ultrasound (DCE-US) for quantification of tumour perfusion. Ultraschall Med 2012;33:344-351.

21. Landmark KE, Johansen PW, Johnson JA, Johansen B, Uran $S$, Skotland T. Pharmacokinetics of perfluorobutane following intravenous bolus injection and continuous infusion of Sonazoid in healthy volunteers and in patients with reduced pulmonary diffusing capacity. Ultrasound Med Biol 2008;34:494-501.

22. Sugimoto K, Moriyasu F, Saito K, Taira J, Saguchi T, Yoshimura N, et al. Comparison of Kupffer-phase Sonazoid-enhanced sonography and hepatobiliary-phase gadoxetic acid-enhanced magnetic resonance imaging of hepatocellular carcinoma and correlation with histologic grading. J Ultrasound Med 2012;31:529-538.

23. Suzuki K, Okuda Y, Ota M, Kojima F, Horimoto M. Diagnosis of hepatocellular carcinoma nodules in patients with chronic liver disease using contrast-enhanced sonography: usefulness of the combination of arterial- and kupffer-phase enhancement patterns. J Ultrasound Med 2015;34:423-433.

24. Shagdarsuren B, Tamai H, Shingaki N, Mori Y, Maeshima S, Nuta J, et al. Contribution of contrast-enhanced sonography with perfluorobutane microbubbles for diagnosis of recurrent hepatocellular carcinoma. J Ultrasound Med 2016;35:1383-1391.

25. Nuta J, Tamai H, Mori Y, Shingaki N, Maeshima S, Shimizu R, et al. Kupffer imaging by contrast-enhanced sonography with perfluorobutane microbubbles is associated with outcomes after radiofrequency ablation of hepatocellular carcinoma. J Ultrasound Med 2016;35:359-371.

26. Lee J, Jeong WK, Lim HK, Kim AY. Focal nodular hyperplasia of the liver: contrast-enhanced ultrasonographic features with Sonazoid. J Ultrasound Med 2018;37:1473-1480.

27. Maruyama H, Takahashi M, Shimada T, Sekimoto T, Kamesaki H, Kanai $F$, et al. Pretreatment microbubble-induced enhancement in hepatocellular carcinoma predicts intrahepatic distant recurrence after radiofrequency ablation. AJR Am J Roentgenol 2013;200:570577.

28. Mandai M, Koda M, Matono T, Nagahara T, Sugihara T, Ueki M, et al. Assessment of hepatocellular carcinoma by contrast-enhanced ultrasound with perfluorobutane microbubbles: comparison with dynamic CT. Br J Radiol 2011;84:499-507.

29. Maruyama H, Takahashi M, Ishibashi H, Yoshikawa M, Yokosuka O. Contrast-enhanced ultrasound for characterisation of hepatic lesions appearing non-hypervascular on $\mathrm{CT}$ in chronic liver diseases. Br J Radiol 2012;85:351-357.

30. Goto E, Masuzaki R, Tateishi R, Kondo Y, Imamura J, Goto T, et al. Value of post-vascular phase (Kupffer imaging) by contrastenhanced ultrasonography using Sonazoid in the detection of hepatocellular carcinoma. J Gastroenterol 2012;47:477-485.

31. Sugimoto K, Moriyasu F, Shiraishi J, Saito K, Taira J, Saguchi T, et al. Assessment of arterial hypervascularity of hepatocellular carcinoma: comparison of contrast-enhanced US and gadoxetate disodiumenhanced MR imaging. Eur Radiol 2012;22:1205-1213.

32. Nuta J, Shingaki N, Ida Y, Shimizu R, Hayami S, Ueno M, et al. Irregular defects in hepatocellular carcinomas during the kupffer phase of contrast-enhanced ultrasonography with perfluorobutane microbubbles: pathological features and metastatic recurrence after surgical resection. Ultrasound Med Biol 2017;43:1829-1836.

33. Joo I, Lee JY, Lee DH, Jeon JH, Kim H, Yi NJ, et al. Contrast-enhanced ultrasound using perfluorobutane-containing microbubbles in the assessment of liver allograft damage: an exploratory prospective study. Ultrasound Med Biol 2017;43:621-628. 
34. Maruyama H, Takahashi M, Sekimoto T, Kamesaki H, Shimada $T$, Kanai $F$, et al. Heterogeneity of microbubble accumulation: a novel approach to discriminate between well-differentiated hepatocellular carcinomas and regenerative nodules. Ultrasound Med Biol 2012;38:383-388.

35. Takahashi M, Maruyama H, Shimada T, Kamezaki H, Sekimoto T, Kanai $F$, et al. Characterization of hepatic lesions ( $\leq 30 \mathrm{~mm})$ with liver-specific contrast agents: a comparison between ultrasound and magnetic resonance imaging. Eur J Radiol 2013;82:75-84.

36. Maruyama H, Takahashi M, Ishibashi H, Okabe S, Yoshikawa M, Yokosuka 0 . Changes in tumor vascularity precede microbubble contrast accumulation deficit in the process of dedifferentiation of hepatocellular carcinoma. Eur J Radiol 2010;75:e102-e106.

37. Numata K, Fukuda H, Miwa H, Ishii T, Moriya S, Kondo M, et al. Contrast-enhanced ultrasonography findings using a perflubutanebased contrast agent in patients with early hepatocellular carcinoma. Eur J Radiol 2014;83:95-102.

38. Kunishi Y, Numata K, Morimoto M, Okada M, Kaneko T, Maeda $S$, et al. Efficacy of fusion imaging combining sonography and hepatobiliary phase MRI with Gd-EOB-DTPA to detect small hepatocellular carcinoma. AJR Am J Roentgenol 2012;198:106114.

39. Tanaka H, lijima H, Higashiura A, Yoh K, Ishii A, Takashima $T$, et al. New malignant grading system for hepatocellular carcinoma using the Sonazoid contrast agent for ultrasonography. J Gastroenterol 2014;49:755-763.

40. Kobayashi K, Maruyama H, Kiyono S, Yokosuka O, Ohtsuka M, Miyazaki $M$, et al. Histology-based assessment of Sonazoidenhanced ultrasonography for the diagnosis of liver metastasis. Ultrasound Med Biol 2017;43:2151-2158.

41. Tada T, Kumada T, Toyoda H, Ito T, Sone Y, Kaneoka Y, et al. Utility of contrast-enhanced ultrasonography with perflubutane for determining histologic grade in hepatocellular carcinoma. Ultrasound Med Biol 2015;41:3070-3078.

42. Tada T, Kumada T, Toyoda H, Ito T, Sone Y, Kaneoka Y, et al. Utility of contrast-enhanced ultrasound with perflubutane for diagnosing the macroscopic type of small nodular hepatocellular carcinomas. Eur Radiol 2014;24:2157-2166.

43. Strobel D, Seitz K, Blank W, Schuler A, Dietrich C, von Herbay A, et al. Contrast-enhanced ultrasound for the characterization of focal liver lesions--diagnostic accuracy in clinical practice (DEGUM multicenter trial). Ultraschall Med 2008;29:499-505.

44. Sugimoto K, Moriyasu F, Saito K, Yoshiara H, Imai Y. Kupffer-phase findings of hepatic hemangiomas in contrast-enhanced ultrasound with Sonazoid. Ultrasound Med Biol 2014;40:1089-1095.

45. Maruyama H, Sekimoto T, Yokosuka O. Role of contrast-enhanced ultrasonography with Sonazoid for hepatocellular carcinoma: evidence from a 10-year experience. J Gastroenterol 2016;51:421433.
46. Inoue T, Hyodo T, Korenaga K, Murakami T, Imai Y, Higaki A, et al. Kupffer phase image of Sonazoid-enhanced US is useful in predicting a hypervascularization of non-hypervascular hypointense hepatic lesions detected on Gd-EOB-DTPA-enhanced MRI: a multicenter retrospective study. J Gastroenterol 2016;51:144-152.

47. Korenaga K, Korenaga M, Furukawa M, Yamasaki T, Sakaida I. Usefulness of Sonazoid contrast-enhanced ultrasonography for hepatocellular carcinoma: comparison with pathological diagnosis and superparamagnetic iron oxide magnetic resonance images. J Gastroenterol 2009;44:733-741.

48. Hatanaka K, Kudo M, Minami Y, Maekawa K. Sonazoid-enhanced ultrasonography for diagnosis of hepatic malignancies: comparison with contrast-enhanced CT. Oncology 2008;75 Suppl 1:42-47.

49. Minami Y, Kudo M. Contrast-enhanced harmonic ultrasound imaging in ablation therapy for primary hepatocellular carcinoma. World J Radiol 2009;1:86-91.

50. Masuzaki R, Shiina S, Tateishi R, Yoshida H, Goto E, Sugioka Y, et al. Utility of contrast-enhanced ultrasonography with Sonazoid in radiofrequency ablation for hepatocellular carcinoma. J Gastroenterol Hepatol 2011;26:759-764.

51. Tanaka M, Nakashima O, Wada Y, Kage M, Kojiro M. Pathomorphological study of Kupffer cells in hepatocellular carcinoma and hyperplastic nodular lesions in the liver. Hepatology 1996;24:807-812.

52. Nanashima A, Tobinaga S, Abo T, Kunizaki M, Takeshita H, Hidaka S, et al. Usefulness of Sonazoid-ultrasonography during hepatectomy in patients with liver tumors: a preliminary study. J Surg Oncol 2011;103:152-157.

53. Eso $Y$, Takai A, Takeda $H$, Matsumoto $T$, Lee $M$, Inuzuka $T$, et al. Sonazoid-enhanced ultrasonography guidance improves the quality of pathological diagnosis in the biopsy of focal hepatic lesions. Eur J Gastroenterol Hepatol 2016;28:1462-1467.

54. Inoue T, Kudo M, Hatanaka K, Takahashi S, Kitai S, Ueda T, et al. Imaging of hepatocellular carcinoma: qualitative and quantitative analysis of postvascular phase contrast-enhanced ultrasonography with Sonazoid. Comparison with superparamagnetic iron oxide magnetic resonance images. Oncology 2008;75 Suppl 1:48-54.

55. Ohama H, Imai Y, Nakashima O, Kogita S, Takamura M, Hori M, et al. Images of Sonazoid-enhanced ultrasonography in multistep hepatocarcinogenesis: comparison with Gd-EOB-DTPA-enhanced MRI. J Gastroenterol 2014;49:1081-1093.

56. Kudo M. Diagnostic imaging of hepatocellular carcinoma: recent progress. Oncology 2011;81 Suppl 1:73-85.

57. Minami Y, Kudo M. Review of dynamic contrast-enhanced ultrasound guidance in ablation therapy for hepatocellular carcinoma. World J Gastroenterol 2011;17:4952-4959.

58. Shiraishi J, Sugimoto K, Moriyasu F, Kamiyama N, Doi K. Computeraided diagnosis for the classification of focal liver lesions by use of contrast-enhanced ultrasonography. Med Phys 2008;35:1734- 
1746.

59. Sugimoto K, Moriyasu F, Kamiyama N, Metoki R, Yamada M, Imai Y, et al. Analysis of morphological vascular changes of hepatocellular carcinoma by microflow imaging using contrast-enhanced sonography. Hepatol Res 2008;38:790-799.

60. Nihonmatsu H, Numata K, Fukuda H, Tanaka K, Ooba M, Maeda S. Low mechanical index contrast mode versus high mechanical index contrast mode: which is a more sensitive method for detecting Sonazoid microbubbles in the liver of normal subjects? J Med Ultrason (2001) 2016;43:211-217.

61. Numata K, Morimoto M, Ogura T, Sugimori K, Takebayashi S, Okada $M$, et al. Ablation therapy guided by contrast-enhanced sonography with Sonazoid for hepatocellular carcinoma lesions not detected by conventional sonography. J Ultrasound Med 2008;27:395-406.

62. Luo W, Numata K, Kondo M, Morimoto M, Sugimori K, Hirasawa $K$, et al. Sonazoid-enhanced ultrasonography for evaluation of the enhancement patterns of focal liver tumors in the late phase by intermittent imaging with a high mechanical index. J Ultrasound Med 2009;28:439-448.

63. Blomley MJ, Albrecht T, Cosgrove DO, Patel N, Jayaram V, ButlerBarnes J, et al. Improved imaging of liver metastases with stimulated acoustic emission in the late phase of enhancement with the US contrast agent SH U 508A: early experience. Radiology 1999;210:409-416.

64. Takizawa K, Numata K, Morimoto M, Kondo M, Nozaki A, Moriya $S$, et al. Use of contrast-enhanced ultrasonography with a perflubutane-based contrast agent performed one day after transarterial chemoembolization for the early assessment of residual viable hepatocellular carcinoma. Eur J Radiol 2013;82:1471-1480.

65. Duisyenbi Z, Numata K, Nihonmatsu H, Fukuda H, Chuma M, Kondo $\mathrm{M}$, et al. Comparison between low mechanical index and high mechanical index contrast modes of contrast-enhanced ultrasonography: evaluation of perfusion defects of hypervascular hepatocellular carcinomas during the post-vascular phase. J Ultrasound Med 2019;38:2329-2338.

66. Numata K, Luo W, Morimoto M, Kondo M, Kunishi Y, Sasaki T, et al. Contrast enhanced ultrasound of hepatocellular carcinoma. World J Radiol 2010;2:68-82.

67. Luo W, Numata K, Morimoto M, Nozaki A, Ueda M, Kondo M, et al. Differentiation of focal liver lesions using three-dimensional ultrasonography: retrospective and prospective studies. World J Gastroenterol 2010;16:2109-2119.

68. Luo W, Numata K, Morimoto M, Kondo M, Takebayashi S, Okada $M$, et al. Focal liver tumors: characterization with 3D perflubutane microbubble contrast agent-enhanced US versus 3D contrastenhanced multidetector CT. Radiology 2009;251:287-295.

69. Luo W, Numata K, Morimoto M, Nozaki A, Nagano Y, Sugimori K, et al. Three-dimensional contrast-enhanced sonography of vascular patterns of focal liver tumors: pilot study of visualization methods.
AJR Am J Roentgenol 2009;192:165-173.

70. Luo W, Numata $K$, Morimoto $M$, Nozaki A, Nagano $Y$, Sugimori $K$, et al. Clinical utility of contrast-enhanced three-dimensional ultrasound imaging with Sonazoid: findings on hepatocellular carcinoma lesions. Eur J Radiol 2009;72:425-431.

71. Luo W, Numata K, Morimoto M, Oshima T, Ueda M, Okada M, et al. Role of Sonazoid-enhanced three-dimensional ultrasonography in the evaluation of percutaneous radiofrequency ablation of hepatocellular carcinoma. Eur J Radiol 2010;75:91-97.

72. Numata K, Fukuda H, Ohto M, Itou R, Nozaki A, Kondou M, et al. Evaluation of the therapeutic efficacy of high-intensity focused ultrasound ablation of hepatocellular carcinoma by threedimensional sonography with a perflubutane-based contrast agent. Eur J Radiol 2010;75:e67-e75.

73. Numata K, Fukuda H, Nihonmatsu H, Kondo M, Nozaki A, Chuma M, et al. Use of vessel patterns on contrast-enhanced ultrasonography using a perflubutane-based contrast agent for the differential diagnosis of regenerative nodules from early hepatocellular carcinoma or high-grade dysplastic nodules in patients with chronic liver disease. Abdom Imaging 2015;40:2372-2383.

74. Sugimori K, Numata K, Okada M, Nihonmatsu H, Takebayashi S, Maeda S, et al. Central vascular structures as a characteristic finding of regenerative nodules using hepatobiliary phase gadolinium ethoxybenzyl diethylenetriaminepentaacetic acidenhanced MRI and arterial dominant phase contrast-enhanced US. J Med Ultrason (2001) 2017:44:89-100.

75. Nishigori S, Numata K, Irie K, Fukuda H, Chuma M, Maeda S. Fusion imaging with contrast-enhanced ultrasonography for evaluating the early therapeutic efficacy of radiofrequency ablation for small hypervascular hepatocellular carcinomas with iso-echoic or unclear margins on conventional ultrasonography. J Med Ultrason (2001) 2018;45:405-415.

76. Kudo M, Ueshima K, Osaki Y, Hirooka M, Imai Y, Aso K, et al. B-Mode Ultrasonography versus contrast-enhanced ultrasonography for surveillance of hepatocellular carcinoma: a prospective multicenter randomized controlled trial. Liver Cancer 2019;8:271-280.

77. Park JH, Park MS, Lee SJ, Jeong WK, Lee JY, Park MJ, et al. Contrast-enhanced US with Perfluorobutane (Sonazoid) used as a surveillance test for hepatocellular carcinoma (HCC) in cirrhosis (SCAN): an exploratory cross-sectional study for a diagnostic trial. BMC Cancer 2017;17:279.

78. Park JH, Park MS, Lee SJ, Jeong WK, Lee JY, Park MJ, et al. Contrastenhanced US with perfluorobutane for hepatocellular carcinoma surveillance: a multicenter diagnostic trial (SCAN). Radiology 2019;292:638-646.

79. Rich NE, Parikh ND, Singal AG. Overdiagnosis: an understudied issue in hepatocellular carcinoma surveillance. Semin Liver Dis 2017;37:296-304. 
80. Atiq O, Tiro J, Yopp AC, Muffler A, Marrero JA, Parikh ND, et al. An assessment of benefits and harms of hepatocellular carcinoma surveillance in patients with cirrhosis. Hepatology 2017;65:11961205.

81. Zhang K, Kokudo N, Hasegawa K, Arita J, Tang W, Aoki T, et al. Detection of new tumors by intraoperative ultrasonography during repeated hepatic resections for hepatocellular carcinoma. Arch Surg 2007;142:1170-1175.

82. Makuuchi M, Hasegawa H, Yamazaki S, Takayasu K, Moriyama $N$. The use of operative ultrasound as an aid to liver resection in patients with hepatocellular carcinoma. World I Surg 1987;11:615621.

83. Torzilli G, Olivari N, Moroni E, Del Fabbro D, Gambetti A, Leoni P, et al. Contrast-enhanced intraoperative ultrasonography in surgery for hepatocellular carcinoma in cirrhosis. Liver Transpl 2004;10(2 Suppl 1):S34-S38.

84. Arita J, Takahashi M, Hata S, Shindoh J, Beck Y, Sugawara Y, et al. Usefulness of contrast-enhanced intraoperative ultrasound using Sonazoid in patients with hepatocellular carcinoma. Ann Surg 2011;254:992-999.

85. Arita J, Ono Y, Takahashi M, Inoue Y, Takahashi Y, Matsueda K, et al. Routine preoperative liver-specific magnetic resonance imaging does not exclude the necessity of contrast-enhanced intraoperative ultrasound in hepatic resection for colorectal liver metastasis. Ann Surg 2015;262:1086-1091.

86. Arita J, Ono Y, Takahashi M, Inoue Y, Takahashi Y, Saiura A. Usefulness of contrast-enhanced intraoperative ultrasound in identifying disappearing liver metastases from colorectal carcinoma after chemotherapy. Ann Surg Oncol 2014;21 Suppl 3:S390-S397.

87. Inoue Y, Arita J, Sakamoto T, Ono Y, Takahashi M, Takahashi Y, et al. Anatomical liver resections guided by 3-dimensional parenchymal staining using fusion indocyanine green fluorescence imaging. Ann Surg 2015;262:105-111.

88. Shindoh J, Seyama Y, Umekita N. Three-dimensional staining of liver segments with an ultrasound contrast agent as an aid to anatomic liver resection. J Am Coll Surg 2012;215:e5-e10.

89. Sugimoto K, Shiraishi J, Moriyasu F, Doi K. Computer-aided diagnosis of focal liver lesions by use of physicians' subjective classification of echogenic patterns in baseline and contrastenhanced ultrasonography. Acad Radiol 2009;16:401-411.

90. Mishima M, Toh U, Iwakuma N, Takenaka M, Furukawa M, Akagi Y. Evaluation of contrast Sonazoid-enhanced ultrasonography for the detection of hepatic metastases in breast cancer. Breast Cancer 2016;23:231-241.

91. Ando Y, Ishigami M, Ishizu Y, Kuzuya T, Honda T, Hirooka Y. Utility of contrast-enhanced ultrasonography with perflubutane in evaluating indications for diagnostic percutaneous tumor biopsy in a case of hepatic sclerosed hemangioma. Clin I Gastroenterol 2018;11:514520.
92. Bioulac-Sage P, Balabaud C, Wanless I. Focal nodular hyperplasia and hepatocellular adenoma. In: Bosman FT, Carneiro F, Hruban $\mathrm{RH}$, Theise ND, eds. WHO classification of tumours of the digestive system. 4th ed. Lyon: International Agency for Research on Cancer, 2010;198-204.

93. Laumonier H, Cailliez H, Balabaud C, Possenti L, Zucman-Rossi J, Bioulac-Sage $\mathrm{P}$, et al. Role of contrast-enhanced sonography in differentiation of subtypes of hepatocellular adenoma: correlation with MRI findings. AJR Am J Roentgenol 2012;199:341-348.

94. Kumagawa M, Matsumoto N, Watanabe $Y$, Hirayama M, Miura T, Nakagawara $\mathrm{H}$, et al. Contrast-enhanced ultrasonographic findings of serum amyloid A-positive hepatocellular neoplasm: does hepatocellular adenoma arise in cirrhotic liver? World J Hepatol 2016;8:1110-1115.

95. Kishina M, Koda M, Tokunaga S, Miyoshi K, Fujise Y, Kato J, et al. Usefulness of contrast-enhanced ultrasound with Sonazoid for evaluating liver abscess in comparison with conventional B-mode ultrasound. Hepatol Res 2015;45:337-342.

96. Yamamoto Y, Fujiwara Y, Yukisawa S, Matsueda K, Katori M, Yamada $K$, et al. Three cases of angiomyolipoma: diagnostic imaging by contrast-enhanced ultrasonography. J Med Ultrason (2001) 2010;37:67-74.

97. Saito M, Seo Y, Yano Y, Miki A, Morinaga Y, Itoh T, et al. Sonazoidenhanced ultrasonography and Ga-EOB-DTPA-enhanced MRI of hepatic inflammatory pseudotumor: a case report. Intern Med 2012;51:723-726.

98. Liver Cancer Study Group of Japan. Classification of primary liver cancer. 6th ed. Tokyo: Kanehara \& Co., Ltd., 2015.

99. Hatanaka K, Kudo M, Minami Y, Ueda T, Tatsumi C, Kitai S, et al. Differential diagnosis of hepatic tumors: value of contrastenhanced harmonic sonography using the newly developed contrast agent, Sonazoid. Intervirology 2008;51 Suppl 1:61-69.

100. Minami Y, Kudo M. Hepatic malignancies: correlation between sonographic findings and pathological features. World J Radiol 2010;2:249-256.

101. Xu HX, Lu MD, Liu GJ, Xie XY, Xu ZF, Zheng YL, et al. Imaging of peripheral cholangiocarcinoma with low-mechanical index contrast-enhanced sonography and SonoVue: initial experience. J Ultrasound Med 2006;25:23-33.

102. Sugimoto K, Shiraishi J, Moriyasu F, Saito K, Doi K. Improved detection of hepatic metastases with contrast-enhanced low mechanical-index pulse inversion ultrasonography during the liver-specific phase of Sonazoid: observer performance study with JAFROC analysis. Acad Radiol 2009;16:798-809.

103. Muhi A, Ichikawa T, Motosugi U, Sou H, Nakajima H, Sano K, et al. Diagnosis of colorectal hepatic metastases: comparison of contrast-enhanced CT, contrast-enhanced US, superparamagnetic iron oxide-enhanced MRI, and gadoxetic acid-enhanced MRI. J Magn Reson Imaging 2011;34:326-335. 
104. Barreiros AP, Piscaglia F, Dietrich CF. Contrast enhanced ultrasound for the diagnosis of hepatocellular carcinoma (HCC): comments on AASLD guidelines. J Hepatol 2012;57:930-932.

105. Shiozawa K, Watanabe M, Ikehara T, Matsukiyo Y, Kikuchi Y, Kaneko $\mathrm{H}$, et al. A case of contiguous primary hepatic marginal zone B-cell lymphoma and hemangioma ultimately diagnosed using contrast-enhanced ultrasonography. Case Rep Oncol 2015;8:50-56.

106. Massarweh NN, El-Serag HB. Epidemiology of hepatocellular carcinoma and intrahepatic cholangiocarcinoma. Cancer Control 2017;24:1073274817729245.

107. Ioannou GN, Splan MF, Weiss NS, McDonald GB, Beretta L, Lee SP. Incidence and predictors of hepatocellular carcinoma in patients with cirrhosis. Clin Gastroenterol Hepatol 2007;5:938-945.

108. Terminology and Diagnostic Criteria Committee, Japan Society of Ultrasonics in Medicine. Ultrasound diagnostic criteria for hepatic tumors. J Med Ultrason (2001) 2014;41:113-123.

109. Kudo M. New sonographic techniques for the diagnosis and treatment of hepatocellular carcinoma. Hepatol Res 2007;37 Suppl 2:S193-S199.

110. Matsui 0. Detection and characterization of hepatocellular carcinoma by imaging. Clin Gastroenterol Hepatol 2005;3(10 Suppl 2):S136-S140.

111. Kojiro M. Histopathology of liver cancers. Best Pract Res Clin Gastroenterol 2005;19:39-62.

112. Park HJ, Choi BI, Lee ES, Park SB, Lee JB. How to differentiate borderline hepatic nodules in hepatocarcinogenesis: emphasis on imaging diagnosis. Liver Cancer 2017;6:189-203.

113. Kutami R, Nakashima Y, Nakashima O, Shiota K, Kojiro M. Pathomorphologic study on the mechanism of fatty change in small hepatocellular carcinoma of humans. J Hepatol 2000;33:282-289.

114. Kudo M. Multistep human hepatocarcinogenesis: correlation of imaging with pathology. J Gastroenterol 2009;44 Suppl 19:112118.

115. Kudo M. Early hepatocellular carcinoma: definition and diagnosis. Liver Cancer 2013;2:69-72.

116. Kudo M. Breakthrough imaging in hepatocellular carcinoma. Liver Cancer 2016;5:47-54.

117. Wilson SR, Lyshchik A, Piscaglia F, Cosgrove D, Jang HJ, Sirlin C, et al. CEUS LI-RADS: algorithm, implementation, and key differences from CT/MRI. Abdom Radiol (NY) 2018;43:127-142.

118. Sarin SK, Philips CA, Kamath PS, Choudhury A, Maruyama H, Nery FG, et al. Toward a comprehensive new classification of portal vein thrombosis in patients with cirrhosis. Gastroenterology 2016;151:574-577.

119. Okuda K, Musha H, Yoshida T, Kanda Y, Yamazaki T. Demonstration of growing casts of hepatocellular carcinoma in the portal vein by celiac angiography: The thread and streaks sign. Radiology
1975;117:303-309.

120. Wang LY, Lin ZY, Chang WY, Chen SC, Chuang WL, Hsieh MY, et al. Duplex pulsed Doppler sonography of portal vein thrombosis in hepatocellular carcinoma. J Ultrasound Med 1991;10:265-269.

121. Tarantino L, Francica G, Sordelli I, Esposito F, Giorgio A, Sorrentino P, et al. Diagnosis of benign and malignant portal vein thrombosis in cirrhotic patients with hepatocellular carcinoma: color Doppler US, contrast-enhanced US, and fine-needle biopsy. Abdom Imaging 2006;31:537-544.

122. Song $Z Z$, Huang $M$, Jiang $T A$, Zhao QY, Yao $L$, Mou $Y$, et al. Diagnosis of portal vein thrombosis discontinued with liver tumors in patients with liver cirrhosis and tumors by contrast-enhanced US: a pilot study. Eur J Radiol 2010;75:185-188.

123. Raza SA, Jang HJ, Kim TK. Differentiating malignant from benign thrombosis in hepatocellular carcinoma: contrast-enhanced ultrasound. Abdom Imaging 2014;39:153-161.

124. Maruyama H, Ishibashi H, Takahashi M, Shimada T, Kamesaki $\mathrm{H}$, Yokosuka O. Prediction of the therapeutic effects of anticoagulation for recent portal vein thrombosis: a novel approach with contrast-enhanced ultrasound. Abdom Imaging 2012;37:431-438.

125. Maruyama H, Takahashi M, Shimada T, Yokosuka O. Emergency anticoagulation treatment for cirrhosis patients with portal vein thrombosis and acute variceal bleeding. Scand J Gastroenterol 2012;47:686-691.

126. Liver Cancer Study Group of Japan. General rules for the clinical and pathological study of primary liver cancer. 6th ed. Tokyo: Kanehara \& Co., Ltd., 2019.

127. Hui AM, Takayama T, Sano K, Kubota K, Akahane M, Ohtomo K, et al. Predictive value of gross classification of hepatocellular carcinoma on recurrence and survival after hepatectomy. J Hepatol 2000;33:975-979.

128. Shimada M, Rikimaru T, Hamatsu T, Yamashita Y, Terashi T, Taguchi $\mathrm{K}$, et al. The role of macroscopic classification in nodular-type hepatocellular carcinoma. Am J Surg 2001;182:177-182.

129. Hatanaka K, Chung H, Kudo M, Haji S, Minami Y, Maekawa K, et al. Usefulness of the post-vascular phase of contrast-enhanced ultrasonography with Sonazoid in the evaluation of gross types of hepatocellular carcinoma. Oncology 2010;78 Suppl 1:53-59.

130. Hatanaka K, Minami Y, Kudo M, Inoue T, Chung H, Haji S. The gross classification of hepatocellular carcinoma: usefulness of contrast-enhanced US. J Clin Ultrasound 2014;42:1-8.

131. Kobayashi T, Aikata H, Hatooka M, Morio K, Morio R, Kan $\mathrm{H}$, et al. Usefulness of combining gadolinium-ethoxybenzyldiethylenetriamine pentaacetic acid-enhanced magnetic resonance imaging and contrast-enhanced ultrasound for diagnosing the macroscopic classification of small hepatocellular carcinoma. Eur Radiol 2015;25:3272-3281.

132. Lipnik AJ, Brown DB. Image-guided percutaneous abdominal mass 
biopsy: technical and clinical considerations. Radiol Clin North Am 2015;53:1049-1059.

133. Sheafor DH, Paulson EK, Simmons CM, DeLong DM, Nelson RC. Abdominal percutaneous interventional procedures: comparison of CT and US guidance. Radiology 1998;207:705-710.

134. Sainani NI, Arellano RS, Shyn PB, Gervais DA, Mueller PR, Silverman SG. The challenging image-guided abdominal mass biopsy: established and emerging techniques 'if you can see it, you can biopsy it'. Abdom Imaging 2013;38:672-696.

135. Kang TW, Lee MW, Song KD, Kim M, Kim SS, Kim SH, et al. Added value of contrast-enhanced ultrasound on biopsies of focal hepatic lesions invisible on fusion imaging guidance. Korean J Radiol 2017;18:152-161.

136. Park HS, Kim YJ, Yu MH, Jung SI, Jeon HJ. Real-time contrastenhanced sonographically guided biopsy or radiofrequency ablation of focal liver lesions using perflurobutane microbubbles (Sonazoid): value of Kupffer-phase imaging. J Ultrasound Med 2015;34:411-421.

137. Park HJ, Lee MW, Lee MH, Hwang J, Kang TW, Lim S, et al. Fusion imaging-guided percutaneous biopsy of focal hepatic lesions with poor conspicuity on conventional sonography. J Ultrasound Med 2013;32:1557-1564.

138. Ahn SJ, Lee JM, Chang W, Lee SM, Kang HJ, Yang HK, et al. Clinical utility of real-time ultrasound-multimodality fusion guidance for percutaneous biopsy of focal liver lesions. Eur J Radiol 2018; 103:76-83.

139. Sparchez Z, Radu P, Kacso G, Sparchez M, Zaharia T, Al Hajjar N. Prospective comparison between real time contrast enhanced and conventional ultrasound guidance in percutaneous biopsies of liver tumors. Med Ultrason 2015;17:456-463.

140. Lorentzen T, Nolsoe CP. The role of US contrast agents in USguided biopsy of focal liver lesions: a pictorial review. Ultrasound Int Open 2019;5:E11-E19.

141. Park MJ, Kim YK, Lee MW, Lee WJ, Kim YS, Kim SH, et al. Small hepatocellular carcinomas: improved sensitivity by combining gadoxetic acid-enhanced and diffusion-weighted MR imaging patterns. Radiology 2012;264:761-770.

142. Kim JE, Kim SH, Lee SJ, Rhim H. Hypervascular hepatocellular carcinoma $1 \mathrm{~cm}$ or smaller in patients with chronic liver disease: characterization with gadoxetic acid-enhanced MRI that includes diffusion-weighted imaging. AJR Am J Roentgenol 2011;196:W758-W765.

143. Lee MW, Kim YJ, Park HS, Yu NC, Jung SI, Ko SY, et al. Targeted sonography for small hepatocellular carcinoma discovered by CT or MRI: factors affecting sonographic detection. AJR Am J Roentgenol 2010;194:W396-W400.

144. Kim PN, Choi D, Rhim H, Rha SE, Hong HP, Lee J, et al. Planning ultrasound for percutaneous radiofrequency ablation to treat small $(\leq 3 \mathrm{~cm})$ hepatocellular carcinomas detected on computed tomography or magnetic resonance imaging: a multicenter prospective study to assess factors affecting ultrasound visibility. J Vasc Interv Radiol 2012;23:627-634.

145. Minami Y, Kudo M, Kawasaki T, Chung H, Ogawa C, Shiozaki $H$. Treatment of hepatocellular carcinoma with percutaneous radiofrequency ablation: usefulness of contrast harmonic sonography for lesions poorly defined with B-mode sonography. AJR Am J Roentgenol 2004;183:153-156.

146. Lee JY, Choi BI, Chung YE, Kim MW, Kim SH, Han JK. Clinical value of CT/MR-US fusion imaging for radiofrequency ablation of hepatic nodules. Eur J Radiol 2012;81:2281-2289.

146. Lee SJ, Won HJ, Kim KW, Shin YM, Kim PN. Value of contrastenhanced sonography of small hepatocellular carcinoma with Sonazoid prior to radiofrequency ablation. J Clin Ultrasound 2017;45:383-390.

148. Minami Y, Kudo M, Hatanaka K, Kitai S, Inoue T, Hagiwara S, et al. Radiofrequency ablation guided by contrast harmonic sonography using perfluorocarbon microbubbles (Sonazoid) for hepatic malignancies: an initial experience. Liver Int 2010;30:759-764.

149. Kudo M, Hatanaka K, Maekawa K. Defect reperfusion imaging, a newly developed novel technology using Sonazoid in the treatment of hepatocellular carcinoma. J Med Ultrasound 2008;16:169-176.

150. Maruyama H, Takahashi M, Ishibashi H, Okugawa H, Okabe S, Yoshikawa $M$, et al. Ultrasound-guided treatments under low acoustic power contrast harmonic imaging for hepatocellular carcinomas undetected by B-mode ultrasonography. Liver Int 2009;29:708-714.

151. Minami $Y$, Kudo M, Chung $H$, Kawasaki T, Yagyu $Y$, Shimono $T$, et al. Contrast harmonic sonography-guided radiofrequency ablation therapy versus B-mode sonography in hepatocellular carcinoma: prospective randomized controlled trial. AJR Am J Roentgenol 2007; 188:489-494.

152. Dohmen T, Kataoka E, Yamada I, Miura K, Ohshima S, Shibuya $T$, et al. Efficacy of contrast-enhanced ultrasonography in radiofrequency ablation for hepatocellular carcinoma. Intern Med 2012;51:1-7.

153. Dietrich CF, Averkiou M, Nielsen MB, Barr RG, Burns PN, Calliada $F$, et al. How to perform Contrast-Enhanced Ultrasound (CEUS). Ultrasound Int Open 2018;4:E2-E15.

154. Nishigaki Y, Hayashi H, Tomita E, Suzuki Y, Watanabe N, Watanabe $S$, et al. Usefulness of contrast-enhanced ultrasonography using Sonazoid for the assessment of therapeutic response to percutaneous radiofrequency ablation for hepatocellular carcinoma. Hepatol Res 2015;45:432-440.

155. Laugesen NG, Nolsoe CP, Rosenberg J. Clinical applications of contrast-enhanced ultrasound in the pediatric eork-up of focal liver lesions and blunt abdominal trauma: a systematic review. Ultrasound Int Open 2017;3:E2-E7. 
156. Inoue T, Kudo M, Hatanaka K, Arizumi T, Takita M, Kitai S, et al. Usefulness of contrast-enhanced ultrasonography to evaluate the post-treatment responses of radiofrequency ablation for hepatocellular carcinoma: comparison with dynamic CT. Oncology 2013;84 Suppl 1:51-57.

157. Numata K, Fukuda $H$, Morimoto $M$, Kondo M, Nozaki A, Oshima $T$, et al. Use of fusion imaging combining contrast-enhanced ultrasonography with a perflubutane-based contrast agent and contrast-enhanced computed tomography for the evaluation of percutaneous radiofrequency ablation of hypervascular hepatocellular carcinoma. Eur J Radiol 2012;81:2746-2753.

158. Lee MW. Fusion imaging of real-time ultrasonography with CT or MRI for hepatic intervention. Ultrasonography 2014;33:227-239.

159. Ahn SJ, Lee JM, Lee DH, Lee SM, Yoon JH, Kim YJ, et al. Realtime US-CT/MR fusion imaging for percutaneous radiofrequency ablation of hepatocellular carcinoma. J Hepatol 2017;66:347-354.

160. Mauri G, Cova L, De Beni S, lerace T, Tondolo T, Cerri A, et al. Realtime US-CT/MRI image fusion for guidance of thermal ablation of liver tumors undetectable with US: results in 295 cases. Cardiovasc Intervent Radiol 2015;38:143-151.

161. Song KD, Lee MW, Rhim H, Cha DI, Chong Y, Lim HK. Fusion imaging-guided radiofrequency ablation for hepatocellular carcinomas not visible on conventional ultrasound. AJR Am J Roentgenol 2013;201:1141-1147.

162. Lee MW, Rhim H, Cha DI, Kim YJ, Choi D, Kim YS, et al. Percutaneous radiofrequency ablation of hepatocellular carcinoma: fusion imaging guidance for management of lesions with poor conspicuity at conventional sonography. AJR Am J Roentgenol 2012;198:1438-1444.

163. Minami T, Minami Y, Chishina $H$, Arizumi T, Takita M, Kitai S, et al. Combination guidance of contrast-enhanced US and fusion imaging in radiofrequency ablation for hepatocellular carcinoma with poor conspicuity on contrast-enhanced US/fusion imaging. Oncology 2014;87 Suppl 1:55-62.

164. Lee MW, Rhim H, Cha DI, Kim YJ, Lim HK. Planning US for percutaneous radiofrequency ablation of small hepatocellular carcinomas (1-3 cm): value of fusion imaging with conventional US and CT/MR images. J Vasc Interv Radiol 2013;24:958-965.

165. Min JH, Lim HK, Lim S, Kang TW, Song KD, Choi SY, et al. Radiofrequency ablation of very-early-stage hepatocellular carcinoma inconspicuous on fusion imaging with B-mode US: value of fusion imaging with contrast-enhanced US. Clin Mol Hepatol 2014;20:61-70.

166. Song KD, Lee MW, Rhim H, Kang TW, Cha DI, Sinn DH, et al. Percutaneous US/MRI fusion-guided radiofrequency ablation for recurrent subcentimeter hepatocellular carcinoma: technical feasibility and therapeutic outcomes. Radiology 2018;288:878886.

167. Lee MW, Lim HK. Management of sub-centimeter recurrent hepatocellular carcinoma after curative treatment: current status and future. World J Gastroenterol 2018;24:5215-5222.

168. Lee MW, Lim HK, Rhim H, Cha DI, Kang TW, Song KD, et al. Percutaneous radiofrequency ablation of small (1-2 $\mathrm{cm})$ hepatocellular carcinomas inconspicuous on B-mode ultrasonographic imaging: usefulness of combined fusion imaging with MRI and contrast-enhanced ultrasonography. Can J Gastroenterol Hepatol 2018;2018:7926923.

169. Rempp H, Loh H, Hoffmann R, Rothgang E, Pan L, Claussen $C D$, et al. Liver lesion conspicuity during real-time MR-guided radiofrequency applicator placement using spoiled gradient echo and balanced steady-state free precession imaging. J Magn Reson Imaging 2014;40:432-439.

170. Anzidei M, Napoli A, Sandolo F, Marincola BC, Di Martino M, Berloco $P$, et al. Magnetic resonance-guided focused ultrasound ablation in abdominal moving organs: a feasibility study in selected cases of pancreatic and liver cancer. Cardiovasc Intervent Radiol 2014;37:1611-1617.

171. Shiozawa K, Watanabe M, Takayama R, Takahashi M, Wakui N, lida $K$, et al. Evaluation of local recurrence after treatment for hepatocellular carcinoma by contrast-enhanced ultrasonography using Sonazoid: comparison with dynamic computed tomography. J Clin Ultrasound 2010;38:182-189.

172. Sugimoto K, Saguchi T, Saito K, Imai Y, Moriyasu F. Hemodynamic changes during balloon-occluded transarterial chemoembolization (B-TACE) of hepatocellular carcinoma observed by contrastenhanced ultrasound. J Med Ultrason (2001) 2014;41:209-215.

173. Sugimoto K, Moriyasu F, Saito K, Rognin N, Kamiyama N, Furuichi $Y$, et al. Hepatocellular carcinoma treated with sorafenib: early detection of treatment response and major adverse events by contrast-enhanced US. Liver Int 2013;33:605-615.

174. Minami Y, Kudo M. Imaging modalities for assessment of treatment response to nonsurgical hepatocellular carcinoma therapy: contrast-enhanced US, CT, and MRI. Liver Cancer 2015;4:106-114.

175. Xia Y, Kudo M, Minami Y, Hatanaka K, Ueshima K, Chung H, et al. Response evaluation of transcatheter arterial chemoembolization in hepatocellular carcinomas: the usefulness of Sonazoidenhanced harmonic sonography. Oncology 2008;75 Suppl 1:99105.

176. Therasse P, Arbuck SG, Eisenhauer EA, Wanders J, Kaplan RS, Rubinstein $L$, et al. New guidelines to evaluate the response to treatment in solid tumors. European Organization for Research and Treatment of Cancer, National Cancer Institute of the United States, National Cancer Institute of Canada. J Natl Cancer Inst 2000;92:205-216.

177. Lencioni R, Llovet JM. Modified RECIST (mRECIST) assessment for hepatocellular carcinoma. Semin Liver Dis 2010;30:52-60.

178. Yoshida K, Hirokawa T, Moriyasu F, Liu L, Liu GJ, Yamada M, et al. 
Arterial-phase contrast-enhanced ultrasonography for evaluating anti-angiogenesis treatment: a pilot study. World I Gastroenterol 2011;17:1045-1050.

179. Shiozawa K, Watanabe M, Ikehara T, Kogame M, Kikuchi Y, Igarashi $Y$, et al. Therapeutic evaluation of sorafenib for hepatocellular carcinoma using contrast-enhanced ultrasonography: Preliminary result. Oncol Lett 2016;12:579-584.

180. Shiozawa K, Watanabe M, Ikehara T, Matsukiyo Y, Kogame M, Shinohara $\mathrm{M}$, et al. Evaluation of sorafenib for hepatocellular carcinoma with low alpha-fetoprotein by arrival time parametric imaging using contrast-enhanced ultrasonography with Sonazoid. Gan To Kagaku Ryoho 2016;43:215-218.

181. Shiozawa K, Watanabe M, Ikehara T, Shimizu R, Shinohara $M$, Igarashi $Y$, et al. Evaluation of sorafenib for advanced hepatocellular carcinoma with low alpha-fetoprotein in arrival time parametric imaging using contrast-enhanced ultrasonography. J Med Ultrason (2001) 2017;44:101-107.

182. Shiozawa K, Watanabe M, Kikuchi Y, Kudo T, Maruyama K, Sumino $Y$. Evaluation of sorafenib for hepatocellular carcinoma by contrast-enhanced ultrasonography: a pilot study. World J Gastroenterol 2012;18:5753-5758.

183. Meier PJ. Transport polarity of hepatocytes. Semin Liver Dis 1988;8:293-307.

184. Reuter SR, Redman HC. Gastrointestinal angiography. Philadelphia, PA: WB Sounders, 1977;306-355.

185. Sumino Y. Contrast-enhanced ultrasound of liver cirrhosis. In: Obara K, ed. Clinical investigation of portal hypertension. Singapore: Springer, 2019;115-126.

186. Wakui N, Takayama R, Kanekawa T, Ichimori M, Otsuka T, Shinohara $\mathrm{M}$, et al. Usefulness of arrival time parametric imaging in evaluating the degree of liver disease progression in chronic hepatitis C infection. J Ultrasound Med 2012;31:373-382.

187. Wakui N, Nagai H, Yoshimine N, Amanuma M, Kobayashi K, Ogino $\mathrm{Y}$, et al. Flash imaging used in the post-vascular phase of contrastenhanced ultrasonography is useful for assessing the progression in patients with hepatitis C virus-related liver disease. Ultrasound Med Biol 2019;45:1654-1662.

188. Miwa H, Numata K, Sugimori K, Kaneko T, Sakamaki K, Ueda M, et al. Differential diagnosis of solid pancreatic lesions using contrastenhanced three-dimensional ultrasonography. Abdom Imaging 2014;39:988-999.

189. Miwa H, Numata K, Sugimori K, Sanga K, Hirotani A, Tezuka S, et al. Differential diagnosis of gallbladder polypoid lesions using contrast-enhanced ultrasound. Abdom Radiol (NY) 2019;44:13671378.

190. Kawai R, Hata J, Manabe N, Imamura H, lida A, Nakatou R, et al. Contrast-enhanced ultrasonography with Sonazoid for diagnosis of gangrenous cholecystitis. J Med Ultrason (2001) 2016;43:193199.
191. Kitano M, Sakamoto H, Matsui U, Ito Y, Maekawa K, von Schrenck $T$, et al. A novel perfusion imaging technique of the pancreas: contrast-enhanced harmonic EUS (with video). Gastrointest Endosc 2008;67:141-150.

192. Dietrich CF, Ignee A, Frey H. Contrast-enhanced endoscopic ultrasound with low mechanical index: a new technique. $Z$ Gastroenterol 2005;43:1219-1223.

193. Fusaroli P, Spada A, Mancino MG, Caletti G. Contrast harmonic echo-endoscopic ultrasound improves accuracy in diagnosis of solid pancreatic masses. Clin Gastroenterol Hepatol 2010;8:629634.

194. Fusaroli P, Napoleon B, Gincul R, Lefort C, Palazzo L, Palazzo M, et al. The clinical impact of ultrasound contrast agents in EUS: a systematic review according to the levels of evidence. Gastrointest Endosc 2016;84:587-596.

195. Gong TT, Hu DM, Zhu Q. Contrast-enhanced EUS for differential diagnosis of pancreatic mass lesions: a meta-analysis. Gastrointest Endosc 2012;76:301-309.

196. Yamashita Y, Shimokawa T, Napoleon B, Fusaroli P, Gincul R, Kudo $M$, et al. Value of contrast-enhanced harmonic endoscopic ultrasonography with enhancement pattern for diagnosis of pancreatic cancer: A meta-analysis. Dig Endosc 2019;31:125-133.

197. Kitano M, Kudo M, Yamao K, Takagi T, Sakamoto H, Komaki T, et al. Characterization of small solid tumors in the pancreas: the value of contrast-enhanced harmonic endoscopic ultrasonography. Am J Gastroenterol 2012;107:303-310.

198. Seicean A, Badea R, Stan-luga R, Mocan T, Gulei I, Pascu 0 . Quantitative contrast-enhanced harmonic endoscopic ultrasonography for the discrimination of solid pancreatic masses. Ultraschall Med 2010;31:571-576.

199. Imazu H, Kanazawa K, Mori N, Ikeda K, Kakutani H, Sumiyama K, et al. Novel quantitative perfusion analysis with contrast-enhanced harmonic EUS for differentiation of autoimmune pancreatitis from pancreatic carcinoma. Scand J Gastroenterol 2012;47:853-860.

200. Saftoiu A, Vilmann P, Dietrich CF, Iglesias-Garcia J, Hocke M, Seicean $A$, et al. Quantitative contrast-enhanced harmonic EUS in differential diagnosis of focal pancreatic masses (with videos). Gastrointest Endosc 2015;82:59-69.

201. Omoto S, Takenaka M, Kitano M, Miyata T, Kamata K, Minaga $\mathrm{K}$, et al. Characterization of pancreatic tumors with quantitative perfusion analysis in contrast-enhanced harmonic endoscopic ultrasonography. Oncology 2017;93 Suppl 1:55-60.

202. Kamata K, Takenaka M, Kitano M, Omoto S, Miyata T, Minaga K, et al. Contrast-enhanced harmonic endoscopic ultrasonography for differential diagnosis of localized gallbladder lesions. Dig Endosc 2018;30:98-106.

203. Choi JH, Seo DW, Choi JH, Park DH, Lee SS, Lee SK, et al. Utility of contrast-enhanced harmonic EUS in the diagnosis of malignant gallbladder polyps (with videos). Gastrointest Endosc 
2013;78:484-493.

204. Imazu H, Mori N, Kanazawa K, Chiba M, Toyoizumi H, Torisu Y, et al. Contrast-enhanced harmonic endoscopic ultrasonography in the differential diagnosis of gallbladder wall thickening. Dig Dis Sci 2014;59:1909-1916.

205. Sugimoto M, Takagi T, Konno N, Suzuki R, Asama H, Hikichi T, et al. The efficacy of contrast-enhanced harmonic endoscopic ultrasonography in diagnosing gallbladder cancer. Sci Rep 2016;6:25848.

206. Leem G, Chung MJ, Park JY, Bang S, Song SY, Chung JB, et al. Clinical value of contrast-enhanced harmonic endoscopic ultrasonography in the differential diagnosis of pancreatic and gallbladder masses. Clin Endosc 2018;51:80-88.

207. Jang JY, Kim MY, Jeong SW, Kim TY, Kim SU, Lee SH, et al. Current consensus and guidelines of contrast enhanced ultrasound for the characterization of focal liver lesions. Clin Mol Hepatol 2013;19:116.

208. Fetzer DT, Rafailidis V, Peterson C, Grant EG, Sidhu P, Barr RG. Artifacts in contrast-enhanced ultrasound: a pictorial essay. Abdom Radiol (NY) 2018;43:977-997.

209. Edey AJ, Ryan SM, Beese RC, Gordon P, Sidhu PS. Ultrasound imaging of liver metastases in the delayed parenchymal phase following administration of Sonazoid using a destructive mode technique (Agent Detection Imaging). Clin Radiol 2008;63:11121120.

210. Miller DL. Overview of experimental studies of biological effects of medical ultrasound caused by gas body activation and inertial cavitation. Prog Biophys Mol Biol 2007;93:314-330.

211. Carstensen EL, Gracewski S, Dalecki D. The search for cavitation in vivo. Ultrasound Med Biol 2000;26:1377-1385.

212. Barnett SB, Duck F, Ziskin M. Recommendations on the safe use of ultrasound contrast agents. Ultrasound Med Biol 2007;33:173174.

213. Dalecki D, Raeman CH, Child SZ, Penney DP, Carstensen EL. Remnants of Albunex nucleate acoustic cavitation. Ultrasound Med Biol 1997;23:1405-1412.

214. Dalecki D, Raeman CH, Child SZ, Cox C, Francis CW, Meltzer $\mathrm{RS}$, et al. Hemolysis in vivo from exposure to pulsed ultrasound. Ultrasound Med Biol 1997;23:307-313.

215. Food Safety Korea [Internet]. Cheongju: Ministry of Food and Drug Safety, 2019 [cited 2020 Apr 21]. Available from: http://nedrug. mfds.go.kr.

216. Pharmaceuticals and Medical Devices Agency [Internet]. Tokyo: Pharmaceuticals and Medical Devices Agency, 2019 [cited 2020 Apr 21]. Available from: http://www.pmda.go.jp/safety/infoservices/drugs/0001.html.

217. Moriyasu F, Itoh K. Efficacy of perflubutane microbubble-enhanced ultrasound in the characterization and detection of focal liver lesions: phase 3 multicenter clinical trial. AJR Am J Roentgenol 2009; 193:86-95.

218. Sonazoid [Internet]. Chicago, IL: GE Healthcare, 2019 [cited 2020 Apr 21]. Available from: http://www3.gehealthcare.co.kr/ko-kr/ products/categories/contrast_media/sonazoid. 